\title{
ROBUST ADAPTIVE FUZZY FILTERS OUTPUT FEEDBACK CONTROL OF STRICT-FEEDBACK NONLINEAR SYSTEMS
}

\author{
Shaocheng TONG, Changliang LiU, Yongming LI \\ Department of Mathematics \\ Liaoning University of Technology, Jinzhou, 121001, People's Republic of China \\ e-mail: jztscesohu.com
}

\begin{abstract}
In this paper, an adaptive fuzzy robust output feedback control approach is proposed for a class of single input single output (SISO) strict-feedback nonlinear systems without measurements of states. The nonlinear systems addressed in this paper are assumed to possess unstructured uncertainties, unmodeled dynamics and dynamic disturbances, where the unstructured uncertainties are not linearly parameterized, and no prior knowledge of their bounds is available. In recursive design, fuzzy logic systems are used to approximate unstructured uncertainties, and K-filters are designed to estimate unmeasured states. By combining backstepping design and a small-gain theorem, a stable adaptive fuzzy output feedback control scheme is developed. It is proven that the proposed adaptive fuzzy control approach can guarantee the all the signals in the closed-loop system are uniformly ultimately bounded, and the output of the controlled system converges to a small neighborhood of the origin. The effectiveness of the proposed approach is illustrated by a simulation example and some comparisons.
\end{abstract}

Keywords: nonlinear systems, adaptive fuzzy control, backstepping, small-gain approach, K-filters.

\section{Introduction}

In the past decade, interest in adaptive control of nonlinear systems has been increasing, and many significant developments have been achieved. As a breakthrough in nonlinear control, adaptive backstepping control was introduced to achieve global stability and asymptotic tracking for a class of nonlinear systems in parametric strictfeedback form by Kanellakopopoulos et al. (1991). Later, the overparametrization problem was successfully eliminated by Kristic et al. (1992) through the tuning function method. In an effort to extend the backstepping control idea to larger classes of nonlinear systems, Kristic et al. (1995) and Qian et al. (2002) studied the adaptive control problem of parametric strict-feedback systems, obtained local stability results, and proposed several adaptive approaches to nonlinear systems with a triangular structure.

To accommodate uncertainties, a robust adaptive backstepping control has been developed for strictfeedback nonlinear systems with time-varying disturbances and static or dynamic uncertainties by Jiang et al. (1998; 1999) (to name a few). The advantages of backstepping methodology include the facts that: (i) global stability can be achieved with ease, (ii) the transient performance can be guaranteed and explicitly analyzed, and (iii) it has the flexibility to avoid unnecessary cancellation of useful nonlinearities compared with feedback linearization techniques. However, these schemes are only suitable for nonlinear systems with nonlinear dynamics models known exactly or with unknown parameters appearing linearly with respect to known nonlinear functions. If that kind of knowledge is not available a priori, these adaptive backstepping controllers cannot be applied.

Fuzzy logic systems have been widely used to model nonlinearities. A fuzzy logic system is a universal approximator which, with the increased size of fuzzy rules, can approximate any nonlinearities with arbitrary precision (Wang, 1994). Based on this capability, fuzzy logic systems are vastly adopted for nonlinear systems identification and control (Chen et al., 1996; Denai et al., 2002; Boukezzoula et al., 2007; Qi et al., 2009). Most of them use fuzzy logic systems as nonlinear models for the underlying nonlinearity. The stability issues for adaptive fuzzy controllers are addressed by Lyapunov functions. However, these adaptive fuzzy controllers are only applied to a relatively simple class of nonlinear systems. The key requirement is that the unknown nonlinearities must satisfy the matching conditions. If the unknown nonlinearities do not satisfy the matching conditions, the adaptive fuzzy controllers mentioned above cannot be implemented. 
To handle the control problem of uncertain nonlinear systems without satisfying matching conditions, in recent years, many backstepping-based adaptive fuzzy controllers have been developed for nonlinear systems in strict-feedback form (Yang et al., 2005; Wang et al., 2007; Zou et al., 2008; Chen et al., 2005; 2007; Tong et al., 2010a; 2010b). Among them, are those for singleinput and single-output (SISO) nonlinear systems (Yang et al., 2005; Wang et al., 2007; Zou et al., 2008; Tong et al., 2010a; 2010b), those for multiple-input and multipleoutput (MIMO) nonlinear systems (Chen et al., 2005; 2007), and the ones for SISO nonlinear systems with dynamics and dynamical disturbances (Tong et al., 2010a; 2010b).

In general, adaptive fuzzy backstepping control can provide a systematic methodology of solving tracking or regulation control problems, where fuzzy systems are used to approximate unknown nonlinear functions. Typically, adaptive fuzzy controllers are constructed recursively in the framework of the traditional backstepping design technique. The main features of these adaptive approaches include the facts that (i) they can deal with those nonlinear systems without satisfying the matching conditions, and (ii) they do not require unknown nonlinear functions being linearly parameterized (Kanellakopopoulos et al., 1991; Kristic et al., 1992; 1995; Qian et al., 2002; Jiang et al., 1998; 1999). Therefore, approximator-based adaptive fuzzy backstepping control has attracted great interest in the intelligent control community.

Despite these efforts regarding adaptive fuzzy backstepping control, the proposed adaptive fuzzy backstepping control methods are all based on the assumption that the states of the systems to be controlled can be measured directly. As noted by Wang (1994), in practice, state variables are often unmeasurable for many nonlinear systems. In such cases, some output feedback control schemes should be applied. It is worth mentioning that, in the case of linear systems, output-feedback control problems can be solved by combining state-feedback controllers with the state observer. However, the separation principle doses not hold for nonlinear systems (Kristic et al., 1995; Qian et al., 2002). Thus, the adaptive output feedback control design is more complex and difficult than the counterpart based on state feedback.

Motivated by the above observations, in this paper, a robust adaptive fuzzy output feedback control approach is proposed for a class of SISO strict-feedback nonlinear systems with modeled dynamics and dynamical disturbances, without measurements of states. Fuzzy logic systems are utilized to approximate unknown nonlinear functions, K-filters are used for estimating unmeasured states, and, combining the backstepping technique and the smallgain theorem, a new stable adaptive fuzzy output feedback robust control scheme is developed. The main advantages of the proposed control scheme are as follows: (i) by de- signing K-filters as a state observer, the proposed control method does not require that all the states of the system be measured directly, which is a common assumption in the existing adaptive fuzzy backstepping controller (Yang et al., 2005; Wang et al., 2007; Tong et al., 2010a; 2010b); (ii) by combining backstepping design with input-to-state practically stability (ISpS) and the small-gain theorem, the proposed control method has a strong robustness to the modeled dynamics and dynamical disturbances, and the stability of entire closed-loop systems can be guaranteed by the small-gain theorem.

It is noted that, in recent years, several adaptive fuzzy backsteping control approaches have also been developed by Yang et al. (2005) and Tong et al. (2010a; 2010b) for some strict-feedback nonlinear systems based on smallgain theorem. However, the approach of Yang et al. (2005) can only control a class of nonlinear systems without unmodeled dynamics or dynamical disturbances and requires that the states of the controlled systems must be measured. Although the approaches of Tong et al. (2010a; 2010b) have addressed the same class of nonlinear systems as this paper, they also require that the states of the nonlinear systems must be measured. Therefore, they cannot be applied to nonlinear systems with unmeasured states.

\section{Problem formulations and some preliminaries}

2.1. Model description and basic assumptions. Consider a class of strict-feedback nonlinear systems with unmodeled dynamics and dynamical disturbances given by the following equations:

$$
\begin{aligned}
\dot{\zeta} & =q(\zeta, y), \\
\dot{x}_{1}= & x_{2}+f_{1,0}(y)+f_{1}(y)+\Delta_{1}(\zeta, y), \\
& \vdots \\
\dot{x}_{n-1}= & x_{n}+f_{n-1,0}(y)+f_{n-1}(y) \\
& +\Delta_{n-1}(\zeta, y), \\
\dot{x}_{n}= & b_{0} \sigma(y) u+f_{n, 0}(y)+f_{n}(y)+\Delta_{n}(\zeta, y), \\
y= & x_{1},
\end{aligned}
$$

where $x=\left[x_{1}, \ldots, x_{n}\right]^{T} \in \mathbb{R}^{n}$ is the state vector, $u \in \mathbb{R}$ is the control input, $y \in \mathbb{R}$ is the output; $\sigma(y)$ is a known smooth nonlinear function $(\sigma(y) \neq 0)$, and $f_{i}(y), 1 \leq i \leq n$ is an unknown smooth nonlinear function; $f_{i, 0}(y), 1 \leq i \leq n$ is a known smooth nonlinear function; $\zeta$ represents unmodeled dynamics and $\Delta_{i}(\zeta, y)$, $1 \leq i \leq n$ represents disturbances related to unmodeled dynamics; $b_{0} \neq 0$ is an unknown constant and the sign of $b_{0}$ is known. In this paper, it is assumed that only $y=x_{1}$ is available for control design.

In the sequel, the following assumptions are imposed on the system (1): 
Assumption 1. (Jiang et al., 1998; 1999) For each $1 \leq$ $i \leq n$, there exists an unknown positive constant $p_{i}^{*}$ such that

$$
\left|\Delta_{i}\right| \leq p_{i}^{*} \psi_{i 1}(|y|)+p_{i}^{*} \psi_{i 2}(|\zeta|)
$$

where $\psi_{i 1}$ and $\psi_{i 2}$ are two known nonnegative smooth functions. Without loss of generality, it is assumed that $\psi_{i 2}(0)=0$.

It is worth mentioning that Assumption 1 implies that the allowed class of uncertainties $\Delta_{i}(x, \varsigma, t)$ satisfies the so-called triangular bounds condition in terms of $x$ and $\varsigma$. The same or similar assumptions can be found in recent works (Jiang et al., 1998; 1999). Such a restriction is crucial in controller design.

Definition 1. (Kristic et al., 1995) A continuous function $\gamma:[0, a) \rightarrow \mathbb{R}_{+}$is said to belong to class $\kappa$ if it is strictly increasing and $\gamma(0)=0$. It is said to belong to class $\kappa_{\infty}$ if $a=\infty$ and $\gamma(r) \rightarrow \infty$ as $r \rightarrow \infty$.

Assumption 2. (Jiang et al., 1998; 1999) Unmodeled dynamics are input-to-state practically stable (ISpS), i.e., the system $\dot{\zeta}=q(\zeta, y)$ has an ISpS Lyapunov function $V_{0}(\zeta)$ such that

$$
\begin{aligned}
\alpha_{1}(|\zeta|) & \leq V_{0}(\zeta) \leq \alpha_{2}(|\zeta|) \\
\frac{\partial V_{0}}{\partial \zeta} q(\zeta, y) & \leq-\alpha_{0}(|\zeta|)+\gamma_{0}(|y|)+d_{0}
\end{aligned}
$$

where $\alpha_{0}, \alpha_{1}, \alpha_{2}$ and $\gamma_{0}$ are $\kappa_{\infty}$-functions defined by Kristic et al. (1995), $d_{0}$ is a nonnegative constant.

Control objectives: The control task is to design an adaptive fuzzy controller using output $y$ only of the form

$$
\dot{\chi}=v(\chi, y), \quad u=\mu(\chi, y),
$$

such that all the signals of the closed-loop systems (1) and (4) are uniformly ultimately bounded. Furthermore, the output can be forced to a small neighborhood of the origin.

Definition 2. (Coddington, 1989) Let $f$ be a function defined for $(x, y)$ in a set $S$. We say that $f$ satisfies locally the Lipschitz condition on $S$ if there exists a constant $M>$ 0 such that

$$
\left|f\left(x, y_{1}\right)-f\left(x, y_{2}\right)\right| \leq M\left|y_{1}-y_{2}\right|
$$

for all $\left(x, y_{1}\right),\left(x, y_{2}\right)$ in $S$. The constant $M$ is called a Lipschitz constant.

Lemma 1. (Jiang et al., 1996) Given the interconnected systems

$$
\begin{aligned}
& \dot{x}_{1}=f_{1}\left(x_{1}, x_{2}, u_{1}\right), \\
& \dot{x}_{2}=f_{2}\left(x_{1}, x_{2}, u_{2}\right),
\end{aligned}
$$

where, for $i=1,2, x_{i} \in \mathbb{R}^{n_{i}}, u_{i} \in \mathbb{R}^{m_{i}}$ and $f_{i}$ : $\mathbb{R}^{n_{1}} \times \mathbb{R}^{n_{2}} \times \mathbb{R}^{m_{i}} \rightarrow \mathbb{R}^{n_{i}}$ locally satisfies the Lipschitz condition.
Assume that, for $i=1,2$, there exists an ISpS-Lyapunov function $V_{i}$ for the $x_{i}$-subsystems such that the following holds:

1. there exist $\kappa_{\infty}$-functions $\vartheta_{i 1}$ and $\vartheta_{i 2}$ such that

$$
\vartheta_{i 1}\left(\left|x_{i}\right|\right) \leq V_{i}\left(x_{i}\right) \leq \vartheta_{i 2}\left(\left|x_{i}\right|\right), \quad \forall x_{i} \in \mathbb{R}^{n_{i}},
$$

2. there exist $\kappa_{\infty}$-function $\alpha_{i}^{\prime}$ and $\kappa$-functions $\chi_{i}, \gamma_{i}$ and some constant $c_{i} \geq 0$ such that, if

$$
V_{1}\left(x_{1}\right) \geq \max \left\{\chi_{1}\left(V_{2}\left(x_{2}\right)\right), \gamma_{1}\left(\left|u_{1}\right|\right)+c_{1}\right\},
$$

then

$$
\nabla V_{1}\left(x_{1}\right) f_{1}\left(x_{1}, x_{2}, u_{1}\right) \leq-\alpha_{1}^{\prime}\left(V_{1}\right)
$$

and, if

$$
V_{2}\left(x_{2}\right) \geq \max \left\{\chi_{2}\left(V_{1}\left(x_{1}\right)\right), \gamma_{2}\left(\left|u_{2}\right|\right)+c_{2}\right\},
$$

then

$$
\nabla V_{2}\left(x_{2}\right) f_{2}\left(x_{1}, x_{2}, u_{2}\right) \leq-\alpha_{2}^{\prime}\left(V_{2}\right) .
$$

A nonlinear small-gain condition is given by Jiang et al. (1996), under which an ISpS-Lyapunov function for the interconnected systems (5)-(6) may be expressed in terms of ISpS-Lyapunov functions for the two subsystems.

Theorem 1. (Jiang et al., 1996) Assume that, for $i=1,2$, the $x_{i}$-subsystems have an ISpS-Lyapunov $V_{i}$ satisfying (7)-(9). If there exists $c_{0} \geq 0$ such that

$$
\chi_{1} \circ \chi_{2}(r)<r, \quad \forall r>c_{0},
$$

then the interconnected system (5)-(6) is ISpS. Furthermore, if $c_{0}=c_{1}=c_{2}=0$, then the system is ISS.

2.2. Fuzzy logic systems and system modeling. A fuzzy logic system (FLS) consists of four parts: a knowledge base, a fuzzifier, a fuzzy inference engine working on fuzzy rules, and a defuzzifier. The knowledge base for an FLS is composed of a collection of fuzzy If-then rules of the following form:

$R^{l}:$ If $x_{1}$ is $F_{1}^{l}$ and $x_{2}$ is $F_{2}^{l}$ and $\ldots$ and $x_{n}$ is $F_{n}^{l}$, then $y$ is $G^{l}, l=1,2, \ldots, N$,

where $x=\left[x_{1}, \ldots, x_{n}\right]^{T}$ and $y$ are the FLS input and output, respectively; $F_{i}^{l}$ and $G^{l}$ are fuzzy sets, associated with the fuzzy functions $\mu_{F_{i}^{l}}\left(x_{i}\right)$ and $\mu_{G^{l}}(y) ; N$ is the rule number.

Through the singleton function, center average defuzzification and product inference, the FLS can be expressed as

$$
y(x)=\frac{\sum_{l=1}^{N} \bar{y}_{l} \prod_{i=1}^{n} \mu_{F_{i}^{l}}\left(x_{i}\right)}{\sum_{l=1}^{N}\left[\prod_{i=1}^{n} \mu_{F_{i}^{l}}\left(x_{i}\right)\right]},
$$


where $\bar{y}_{l}=\max _{y \in R} \mu_{G^{l}}(y)$.

Define the fuzzy basis functions as

$$
\varphi_{l}=\frac{\prod_{i=1}^{n} \mu_{F_{i}^{l}}\left(x_{i}\right)}{\sum_{l=1}^{N}\left(\prod_{i=1}^{n} \mu_{F_{i}^{l}}\left(x_{i}\right)\right)} .
$$

If we let $\theta=\left[\bar{y}_{1}, \bar{y}_{2}, \ldots, \bar{y}_{N}\right]^{T}=\left[\bar{\theta}_{1}, \bar{\theta}_{2}, \ldots, \bar{\theta}_{N}\right]^{T}$ and $\varphi^{T}(x)=\left[\varphi_{1}(x), \ldots, \varphi_{N}(x)\right]$ then the FLS (11) can be rewritten as

$$
y(x)=\theta^{T} \varphi(x) .
$$

It has been proved that the fuzzy logic system (12) can approximate any continuous function $f(x)$ over a compact set $\Omega \subset \mathbb{R}^{q}$ to any arbitrary accuracy as

$$
f(x)=\theta^{* T} \varphi(x)+\varepsilon(x), \quad \forall x \in \Omega,
$$

where $\theta^{*}$ is an ideal constant parameter, and $\varepsilon(x)$ is the fuzzy minimums approximation error, which is defined by Wang (1994) as

$$
\theta^{*}=\arg \min _{\theta \in U}\left\{\sup _{y \in \Omega}\left|f(x)-\theta^{T} \varphi(x)\right|\right\} .
$$

By employing the FLS to approximate the unknown smooth function $f_{i}(y)$ in (1) and assuming that

$$
f_{i}(y)=\theta_{i}^{* T} \varphi_{i}(y)+\varepsilon_{i}(y)
$$

denote the fuzzy minimums approximation error vector as $\varepsilon(y)=\left[\begin{array}{lll}\varepsilon_{1}(y) & \ldots & \varepsilon_{n}(y)\end{array}\right]^{T}$.

Assumption 3. The fuzzy minimum approximation error vector $\varepsilon(y)$ satisfies $\|\varepsilon(y)\| \leq \beta$, where $\beta$ is an unknown positive constant, and $\|\cdot\|$ represents the 2-norm of a vector.

By substituting (14) into (1), the system (1) can be expressed as

$$
\begin{aligned}
\dot{\zeta}= & q(\zeta, y), \\
\dot{x}_{1}= & x_{2}+f_{1,0}(y)+\theta_{1}^{* T} \varphi_{1}(y) \\
& +\varepsilon_{1}(y)+\Delta_{1}(\zeta, y), \\
\vdots & \\
\dot{x}_{n-1}= & x_{n}+f_{n-1,0}(y)+\theta_{n-1}^{* T} \varphi_{n-1}(y) \\
& +\varepsilon_{n-1}(y)+\Delta_{n-1}(\zeta, y), \\
\dot{x}_{n}= & b_{0} \sigma(y) u+f_{n, 0}(y)+\theta_{n}^{* T} \varphi_{n}(y) \\
& +\varepsilon_{n}(y)+\Delta_{n}(\zeta, y), \\
y= & x_{1} .
\end{aligned}
$$

Rewrite (15) as

$$
\begin{aligned}
\dot{\zeta}= & q(\zeta, y), \\
\dot{x}= & A x+f_{0}(y)+\Phi^{T}(y) \theta+\varepsilon(y) \\
& +\Delta+\left[\begin{array}{ll}
0 & b_{0},
\end{array}\right]^{T} \sigma(y) u \\
y= & C_{1}^{T} x,
\end{aligned}
$$

where

$$
\begin{aligned}
& A=\left[\begin{array}{cc}
0 & \\
\vdots & I_{n-1} \\
0 & \cdots 0
\end{array}\right], \\
& f_{0}(y)=\left[\begin{array}{c}
f_{1,0}(y) \\
\vdots \\
f_{n, 0}(y)
\end{array}\right] \text {, } \\
& \Phi^{T}(y)=\left[\begin{array}{ccc}
\varphi_{1}^{T}(y) & & \\
& \ddots & \\
& & \varphi_{n}^{T}(y)
\end{array}\right]_{n \times l} \text {, } \\
& l=l_{1}+\cdots+l_{n}, \\
& C_{1}=[1,0, \ldots, 0]^{T} \text {, } \\
& \theta^{T}=\left[\begin{array}{lll}
\theta_{1}^{*} & \cdots & \theta_{n}^{*}
\end{array}\right]_{1 \times l}, \\
& \Delta=\left[\begin{array}{lll}
\Delta_{1} & \cdots & \Delta_{n}
\end{array}\right]^{T} .
\end{aligned}
$$

The system (16) is further rewritten as

$$
\begin{aligned}
\dot{\zeta} & =q(\zeta, y), \\
\dot{x} & =A x+f_{0}(y)+G^{T}(y, u) \vartheta+\varepsilon(y)+\Delta, \\
y & =C_{1}^{T} x,
\end{aligned}
$$

where

$$
\begin{aligned}
\vartheta & =\left[\begin{array}{c}
b_{0} \\
\theta
\end{array}\right]_{(l+1) \times 1}, \\
G^{T}(y, u) & =\left[\left[\begin{array}{c}
0_{(n-1) \times 1} \\
1
\end{array}\right] \sigma(y) u, \Phi^{T}(y)\right] .
\end{aligned}
$$

Choose a vector $k=\left[k_{1}, \cdots, k_{n}\right]^{T}$ so that the matrix $A_{0}=A-k C_{1}^{T}$ is a strict Hurwitz matrix, i.e., given a positive definite matrix $Q=Q^{T}>0$, there exists a positive definite matrix $P=P^{T}>0$ such that

$$
P A_{0}+A_{0}^{T} P=-Q .
$$

\section{Adaptive fuzzy controller design and stability analysis}

Note that in the system (1) or (16), the states $x_{2}, x_{3}, \ldots$, $x_{n}$ are an unmeasured, $b_{0}$ and $\theta$ are an unknown constant and unknown parameter vector, respectively. Thus, the states of the system (1), $b_{0}$ and $\theta$ should be estimated by using the filters given by Kristic et al. (1995) as well as Ye (2001). Define the virtual state estimate as

$$
\hat{x}=\xi+\Omega^{T} \vartheta .
$$

According to Kristic et al. (1995) and from (18), the K-filters may be defined as follows:

$$
\begin{aligned}
\dot{\xi} & =A_{0} \xi+k y+f_{0}(y), \\
\dot{\Omega}^{T} & =A_{0} \Omega^{T}+G^{T}(y, u) .
\end{aligned}
$$


Note that the parameter vector $\vartheta$ is unknown, and, as such, it cannot be used in control design. Therefore, an estimate $\hat{\vartheta}$ of the parameter vector $\vartheta$ need to be obtained later. On the other hand, the virtual state estimate defined by (20) is not used in control design, and the actual state estimate should be $\hat{\hat{x}}=\xi+\Omega^{T} \hat{\vartheta}$. Denote by $v_{0}$ the first column of $\Omega^{T}$. The vector $v_{0}$ is governed by

$$
\dot{v}_{0}=A_{0} v_{0}+C_{n} \sigma(y) u,
$$

where $C_{n}=[0, \cdots, 0,1]^{T}$. In view of (21) and (22), $\Omega$ is expressed as

$$
\Omega^{T}=\left[v_{0}, \Xi\right] .
$$

From (21), one obtains

$$
\dot{\Xi}=A_{0} \Xi+\Phi^{T}(y) .
$$

Define the observation error vector $e$ as

$$
e=\left[e_{1}, e_{2}, \cdots, e_{n}\right]^{T}=\frac{x-\hat{x}}{p^{*}},
$$

where $p^{*}=\max \left\{p_{i}^{*}, p_{i}^{* 2}, 1 ; 1 \leq i \leq n\right\}$ is an unknown constant. The time derivative of $e$ can be expressed as

$$
\dot{e}=A_{0} e+\frac{\varepsilon(y)+\Delta}{p^{*}} .
$$

From the second equation in (16), one obtains

$$
\dot{y}=x_{2}+f_{1,0}(y)+\theta_{1}^{* T} \varphi_{1}(y)+\varepsilon_{1}(y)+\Delta_{1} .
$$

Since $x_{2}$ is unavailable, it is replaced by available filter signals. From (18), one has

$$
\begin{aligned}
x & =\xi+\Omega^{T} \vartheta+x-\hat{x} \\
& =\xi+\Omega^{T} \vartheta+p^{*} e .
\end{aligned}
$$

Therefore, using (28), $x_{2}$ is expressed as

$$
\begin{aligned}
x_{2} & =\xi_{2}+\Omega_{(2)}^{T} \vartheta+p^{*} e_{2} \\
& =b_{0} v_{0,2}+\xi_{2}+\left[0, \Xi_{(2)}\right] \vartheta+p^{*} e_{2},
\end{aligned}
$$

where $\Omega_{(2)}^{T}$ and $\Xi_{(2)}$ are the second rows of $\Omega^{T}$ and $\Xi$, respectively.

Substituting (29) into (27) yields

$$
\dot{y}=b_{0} v_{0,2}+\xi_{2}+f_{1,0}(y)+\bar{\omega}^{T} \vartheta+p^{*} e_{2}+\varepsilon_{1}(y)+\Delta_{1},
$$

where the "regressor" $\omega$ and the "truncated regressor" $\bar{\omega}$ are defined by Kristic et al. (1995) as follows

$$
\begin{aligned}
& \omega=\left[v_{0,2}, \Phi_{(1)}^{T}(y)+\Xi_{(2)}\right]^{T}, \\
& \bar{\omega}=\left[0, \Phi_{(1)}^{T}(y)+\Xi_{(2)}\right]^{T} .
\end{aligned}
$$

From (22), we obtain

$$
\begin{aligned}
\dot{v}_{0, i} & =v_{0, i+1}-k_{i} v_{0,1}, \quad i=2, \ldots, n,-1, \\
\dot{v}_{0, n} & =\sigma(y) u-k_{n} v_{0,1} .
\end{aligned}
$$

Define a change of coordinates as

$$
\begin{aligned}
z_{1} & =y \lambda^{\prime}\left(y^{2}\right), \\
z_{i} & =v_{0, i}-\pi_{i-1}, \quad i=2, \cdots, n,
\end{aligned}
$$

where $\lambda^{\prime}\left(y^{2}\right)$ is the derivative of a smooth class $\kappa_{\infty}$-function $\lambda\left(y^{2}\right)$, and $\lambda^{\prime}\left(y^{2}\right) \neq 0$, which will be chosen later.

After the above preparations, adaptive fuzzy backstepping control design is given by the following procedures.

Step 0: Consider the following Lyapunov function:

$$
V_{0}=e^{T} P e .
$$

The time derivative of $V_{0}$ along (26) is

$$
\dot{V}_{0}=e^{T}\left(A_{0}^{T} P+P A_{0}\right) e+\frac{2}{p^{*}} e^{T} P(\varepsilon+\Delta) .
$$

By Assumption 1 and Young's inequality $2 a b \leq a^{2}+b^{2}$ and $p^{*} \geq 1$, we have

$$
\begin{aligned}
\frac{2}{p^{*}} e^{T} P \Delta & \leq \frac{2}{p^{*}}\|e\|\|P\|\|\Delta\| \\
& \leq \frac{2}{p^{*}} \sum_{i=1}^{n}\|e\|\|P\|\left|\Delta_{i}\right| \\
& \leq 2\|e\|\|P\|\left(\sum_{k=1}^{n} \psi_{k 1}(|y|)+\sum_{k=1}^{n} \psi_{k 2}(|\zeta|)\right),
\end{aligned}
$$

$$
\begin{aligned}
2\|e\|\|P\| \sum_{k=1}^{n} \psi_{k 1}(|y|) & \\
& \leq\|e\|^{2}+\|P\|^{2}\left(\sum_{k=1}^{n} \psi_{k 1}(|y|)\right)^{2} .
\end{aligned}
$$

Since $\psi_{i 1}$ is a smooth function, using the same proof of Jiang (1999), we get

$$
\left(\sum_{k=1}^{n} \psi_{k 1}(|y|)\right)^{2} \leq y^{2} \phi_{1}(y)+d_{\psi}^{0},
$$

where $\phi_{1}$ is a smooth nonnegative function, and $d_{\psi}^{0}=$ $\left(\sum_{i=1}^{n} \psi_{i 1}(0)\right)^{2}$ is a constant.

Substituting (41) into (40) yields

$$
\begin{aligned}
& 2\|e\|\|P\| \sum_{k=1}^{n} \psi_{k 1}(|y|) \\
& \quad \leq\|e\|^{2}+\|P\|^{2} y^{2} \phi_{1}(y)+\|P\|^{2} d_{\psi}^{0} .
\end{aligned}
$$

Using Young's inequality, we have

$$
\begin{aligned}
& 2\|e\|\|P\| \sum_{k=1}^{n} \psi_{k 2}(|\zeta|) \\
& \quad \leq\|e\|^{2}+\|P\|^{2}\left(\sum_{k=1}^{n} \psi_{k 2}(|\zeta|)\right)^{2},
\end{aligned}
$$


$\frac{2}{p^{*}} e^{T} P \varepsilon \leq 2\|e\|\|P\|\|\varepsilon\| \leq\|e\|^{2}+\|P\|^{2} \beta^{2}$.

Substituting (42)-(44) into (38), we obtain

$$
\begin{aligned}
\dot{V}_{1} \leq & -\left[\lambda_{\min }(Q)-3\right]\|e\|^{2} \\
& +\|P\|^{2}\left(\sum_{k=1}^{n} \psi_{k 2}(|\zeta|)\right)^{2}+\|P\|^{2} y^{2} \phi_{1}(y) \\
& +\|P\|^{2} \beta^{2}+\|P\|^{2} d_{\psi}^{0} .
\end{aligned}
$$

Step 1: Consider the following Lyapunov function:

$$
\begin{aligned}
V_{1}= & V_{0}+\frac{1}{2} \lambda\left(y^{2}\right)+\frac{1}{2} \tilde{\vartheta}^{T} \Gamma^{-1} \tilde{\vartheta} \\
& +\frac{1}{2} \gamma_{1}^{-1} \tilde{\beta}^{2}+\frac{1}{2} \gamma_{2}^{-1} \tilde{p}^{2}+\frac{1}{2} \gamma_{3}^{-1}\left|b_{0}\right| \tilde{\kappa}^{2},
\end{aligned}
$$

where $\Gamma=\Gamma^{T}>0, \gamma_{1}>0, \gamma_{2}>0$ and $\gamma_{3}>0$ are design constants; $\tilde{\vartheta}=\vartheta-\hat{\vartheta}, \tilde{\beta}=\beta-\hat{\beta}, \tilde{p}=p-\hat{p}$ and $\tilde{\kappa}=\kappa-\hat{\kappa} ; \hat{\vartheta}, \hat{\beta}, \hat{p}$ and $\hat{\kappa}$ are the estimates of $\vartheta, \beta, p$ and $\kappa$, respectively. Here $\kappa=b_{0}^{-1}$ is an unknown constant. Define $\pi_{1}=\hat{\kappa} \bar{\pi}_{1}$, where $\bar{\pi}_{1}$ is a stabilizing function to be designed later.

The time derivative of $V_{1}$ along (30) is

$$
\begin{aligned}
\dot{V}_{1}= & \dot{V}_{0}+y \lambda^{\prime}\left(y^{2}\right) \dot{y}-\tilde{\vartheta}^{T} \Gamma^{-1} \dot{\hat{\vartheta}} \\
& -\gamma_{1}^{-1} \tilde{\beta} \dot{\hat{\beta}}-\gamma_{2}^{-1} \tilde{p} \dot{\hat{p}}-\gamma_{3}^{-1}\left|b_{0}\right| \tilde{\kappa} \dot{\hat{\kappa}} \\
= & \dot{V}_{0}+y \lambda^{\prime}\left(y^{2}\right)\left(b_{0} v_{0,2}+\xi_{2}\right. \\
& +f_{1,0}(y)+\bar{\omega}^{T} \vartheta+p^{*} e_{2} \\
& \left.+\varepsilon_{1}(y)+\Delta_{1}\right)-\tilde{\vartheta} \Gamma^{-1} \dot{\hat{\vartheta}}-\gamma_{1}^{-1} \tilde{\beta} \dot{\hat{\beta}} \\
& -\gamma_{2}^{-1} \tilde{p} \dot{\hat{p}}-\gamma_{3}^{-1}\left|b_{0}\right| \tilde{\kappa} \dot{\hat{\kappa}} .
\end{aligned}
$$

Substituting (35) into (47) results in

$$
\begin{aligned}
\dot{V}_{1}= & \dot{V}_{0}+z_{1}\left(b_{0} v_{0,2}+\xi_{2}+f_{1,0}(y)\right. \\
& \left.+\bar{\omega}^{T} \vartheta+p^{*} e_{2}+\varepsilon_{1}(y)+\Delta_{1}\right) \\
& -\tilde{\vartheta}^{T} \Gamma^{-1} \dot{\hat{\vartheta}}-\gamma_{1}^{-1} \tilde{\beta} \hat{\hat{\beta}}-\gamma_{2}^{-1} \tilde{p} \dot{\hat{p}} \\
& -\gamma_{3}^{-1}\left|b_{0}\right| \tilde{\kappa} \dot{\hat{\kappa}} \\
= & \dot{V}_{0}+b_{0} z_{1} z_{2}-z_{1} b_{0} \tilde{\kappa} \bar{\pi}_{1} \\
& +z_{1}\left(\bar{\pi}_{1}+\xi_{2}+f_{1,0}(y)+\bar{\omega}^{T} \vartheta\right) \\
& +y \lambda^{\prime}\left(y^{2}\right)\left(p^{*} e_{2}+\varepsilon_{1}(y)+\Delta_{1}\right) \\
& -\tilde{\vartheta}^{T} \Gamma^{-1} \dot{\hat{\vartheta}}-\gamma_{1}^{-1} \tilde{\beta} \dot{\hat{\beta}} \\
& -\gamma_{2}^{-1} \tilde{p} \dot{\hat{p}}-\gamma_{3}^{-1}\left|b_{0}\right| \tilde{\kappa} \dot{\hat{\kappa}} .
\end{aligned}
$$

Using Assumption 1 and Young's inequality, we have

$$
\begin{aligned}
& y \lambda^{\prime}\left(y^{2}\right)\left(p^{*} e_{2}+\Delta_{1}\right) \\
& \leq p^{*}\left|y \lambda^{\prime}\left(y^{2}\right)\right|\left|e_{2}\right|+\left|y \lambda^{\prime}\left(y^{2}\right)\right|\left|\Delta_{1}\right| \\
& \leq p^{*}\left|y \lambda^{\prime}\left(y^{2}\right)\right|\left|e_{2}\right|+p_{1}^{*}\left|y \lambda^{\prime}\left(y^{2}\right)\right|\left(\psi_{11}(|y|)\right. \\
& \left.\quad-\psi_{11}(|0|)\right)+p_{1}^{*}\left|y \lambda^{\prime}\left(y^{2}\right)\right| \psi_{12}(|\zeta|) \\
& \quad+p_{1}^{*}\left|y \lambda^{\prime}\left(y^{2}\right)\right| \psi_{11}(|0|)
\end{aligned}
$$

$$
\begin{aligned}
& \leq\left|e_{2}\right|^{2}+\frac{p_{1}^{* 2}}{4}\left(y \lambda^{\prime}\left(y^{2}\right)\right)^{2} \\
& +p_{1}^{*} y^{2} \lambda^{\prime}\left(y^{2}\right) \bar{\psi}_{11}(|y|) \\
& +\frac{p_{1}^{* 2}}{2}\left(y \lambda^{\prime}\left(y^{2}\right)\right)^{2}+\psi_{12}^{2}(|\zeta|)+\psi_{11}^{2}(0),
\end{aligned}
$$

where $\bar{\psi}_{11}(|y|)=\int_{0}^{1} \psi_{11}^{\prime}(s|y|) \mathrm{d} s$.

Using the proof of Jiang (1999), given any $d_{11}>0$, there exists a smooth function $\hat{\psi}_{11}$ with $\hat{\psi}_{11}(0)=0$, such that

$$
|y| \bar{\psi}_{11}(|y|) \leq y \hat{\psi}_{11}(y)+d_{11}, \quad \forall y \in \mathbb{R},
$$

Therefore, (49) can be rewritten as

$$
\begin{aligned}
& y \lambda^{\prime}\left(y^{2}\right)\left(p^{*} e_{2}+\Delta_{1}\right) \\
& \leq\|e\|^{2}+p \phi_{11}(y)\left(y \lambda^{\prime}\left(y^{2}\right)\right)^{2} \\
& +\psi_{12}^{2}(|\zeta|)+d_{11}^{2}+\psi_{11}^{2}(0),
\end{aligned}
$$

where $p=\left(p^{*}\right)^{2}$ and

$$
\phi_{11}(y)=1+1 /\left(2 \lambda^{\prime}\left(y^{2}\right)\right)+1 /\left(2 \lambda^{\prime}\left(y^{2}\right)\right) \hat{\psi}_{11}^{2}(y)
$$

is a smooth nonnegative function.

Note that, for $\forall \varsigma>0$, the following inequality holds:

$$
|r|-r \tanh (r / \varsigma) \leq 0.2785 \varsigma .
$$

By (51), one has

$$
\begin{aligned}
& \left|\varepsilon_{1}(y) z_{1}\right|-z_{1} \eta_{1} \beta \tanh \left(\frac{z_{1} \eta_{1}}{\varsigma}\right) \\
& \quad \leq \beta\left(\left|z_{1}\right|-z_{1} \eta_{1} \tanh \left(\frac{z_{1} \eta_{1}}{\varsigma}\right)\right) \\
& \quad \leq 0.2785 \varsigma \beta=\varsigma^{\prime},
\end{aligned}
$$

where $\varsigma$ is an arbitrary small constant and $\eta_{1}=-1$.

Substituting (45), (50) and (52) into (48) yields

$$
\begin{aligned}
\dot{V}_{1} \leq & -\left[\lambda_{\min }(Q)-4\right]\|e\|^{2}+z_{1}\left(\bar{\pi}_{1}+\xi_{2}\right. \\
& +f_{1,0}(y)+\bar{\omega} \hat{\vartheta}+\frac{\|P\|^{2}}{\lambda^{\prime}} y \phi_{1}(y) \\
& \left.+\hat{p} \phi_{11}(y) z_{1}+\eta_{1} \hat{\beta} \tanh \left(\frac{z_{1} \eta_{1}}{\varsigma}\right)\right) \\
& +b_{0} z_{1} z_{2}+\tilde{\vartheta} \tilde{\vartheta}^{T}\left(\bar{\omega} z_{1}-\Gamma^{-1} \dot{\hat{\vartheta}}\right) \\
& +\tilde{\beta}\left(z_{1} \eta_{1} \beta \tanh \left(\frac{z_{1} \eta_{1}}{\varsigma}\right)-\gamma_{1}^{-1} \dot{\hat{\beta}}\right) \\
& +\tilde{p}\left(\phi_{11}(y) z_{1}^{2}-\gamma_{2}^{-1} \hat{\hat{\hat{p}}}\right)-\tilde{\kappa}\left(z_{1} b_{0} \bar{\pi}_{1}\right. \\
& \left.+\gamma_{3}^{-1}\left|b_{0}\right| \dot{\hat{\kappa}}\right)+\|P\|^{2}\left(\sum_{k=1}^{n} \psi_{k 2}(|\zeta|)\right)^{2} \\
& +\psi_{12}^{2}(|\zeta|)+\|P\|^{2} \beta^{2}+\|P\|^{2} d_{\psi}^{0} \\
& +\psi_{11}^{2}(0)+\varsigma^{\prime}+d_{11}^{2} .
\end{aligned}
$$


Choose the stabilizing control function $\bar{\pi}_{1}$, tuning functions and parameters adaptation laws as

$$
\begin{aligned}
\bar{\pi}_{1} & =-y \rho\left(y^{2}\right)-\xi_{2}-f_{1,0}(y)-\bar{\omega} \hat{\vartheta} \\
& -\frac{\|P\|^{2}}{\lambda^{\prime}} y \varphi_{1}(y)-\hat{p} \phi_{11}(y) z_{1} \\
& -\eta_{1} \hat{\beta} \tanh \left(\frac{z_{1} \eta_{1}}{\varsigma}\right), \\
\tau_{1} & =\bar{\omega} z_{1}, \\
\sigma_{1} & =z_{1} \eta_{1} \beta \tanh \left(\frac{z_{1} \eta_{1}}{\varsigma}\right), \\
\bar{\lambda}_{1} & =\phi_{11}(y) z_{1}^{2}, \\
\dot{\hat{\kappa}} & =-\gamma_{3}\left(\operatorname{sgn}\left(b_{0}\right) \bar{\pi}_{1} z_{1}+\mu \hat{\kappa}\right),
\end{aligned}
$$

where $\rho\left(y^{2}\right)$ is a smooth non-decreasing function with $\rho(0)>0$, and $\mu>0$ is a design parameter. Substituting (54)-(58) into (53) yields

$$
\begin{aligned}
\dot{V}_{1} \leq & -\left[\lambda_{\min }(Q)-4\right]\|e\|^{2}-z_{1} y \rho\left(y^{2}\right) \\
& +b_{0} z_{1} z_{2}+\tilde{\vartheta}^{T}\left(\tau_{1}-\Gamma^{-1} \dot{\hat{\vartheta}}\right) \\
& +\tilde{\beta}\left(\sigma_{1}-\gamma_{1}^{-1 i} \beta\right)+\tilde{p}\left(\bar{\lambda}_{1}-\gamma_{2}^{-1} \dot{\hat{p}}\right) \\
& +\mu \tilde{\kappa} \hat{\kappa}+\|P\|^{2}\left(\sum_{k=1}^{n} \psi_{k 2}(|\zeta|)\right)^{2} \\
& +\psi_{12}^{2}(|\zeta|)+\|P\|^{2} \beta^{2}+\|P\|^{2} d_{\psi}^{0} \\
& +\varsigma^{\prime}+\psi_{11}^{2}(0)+d_{11}^{2} .
\end{aligned}
$$

Step 2: The time derivative of $z_{2}$ along (36) is

$$
\begin{aligned}
\dot{z}_{2}= & v_{0,3}-k_{2} v_{0,1}-\frac{\partial \pi_{1}}{\partial y}\left(\xi_{2}+f_{1,0}(y)\right. \\
& \left.+\omega^{T} \vartheta+p^{*} e_{2}+\Delta_{1}+\varepsilon_{1}(y)\right) \\
& -\frac{\partial \pi_{1}}{\partial \xi}\left(A_{0} \xi+k y\right)-\frac{\partial \pi_{1}}{\partial \Xi}\left(A_{0} \Xi+\Phi^{T}(y)\right) \\
& -\frac{\partial \pi_{1}}{\partial v_{0}} \dot{v}_{0}-\frac{\partial \pi_{1}}{\partial \kappa} \dot{\kappa}-\frac{\partial \pi_{1}}{\partial \hat{\vartheta}} \Gamma\left(\tau_{1}-\mu \hat{\vartheta}\right) \\
& -\frac{\partial \pi_{1}}{\partial \hat{\beta}} \gamma_{1}\left(\sigma_{1}-\mu \hat{\beta}\right)-\frac{\partial \pi_{1}}{\partial \hat{p}} \gamma_{2}\left(\bar{\lambda}_{1}-\mu \hat{p}\right) \\
& \left.-\frac{\partial \pi_{1}}{\partial \hat{\vartheta}} \dot{(} \vartheta-\Gamma \tau_{1}+\Gamma \mu \hat{\vartheta}\right) \\
& \left.-\frac{\partial \pi_{1}}{\partial \hat{\beta}} \dot{(} \beta-\gamma_{1} \sigma_{1}+\gamma_{1} \mu \hat{\beta}\right) \\
& \left.-\frac{\partial \pi_{1}}{\partial \hat{p}} \dot{(p}-\gamma_{2} \bar{\lambda}_{1}+\gamma_{2} \mu \hat{p}\right) .
\end{aligned}
$$

Consider the Lyapunov function

$$
V_{2}=V_{1}+\frac{1}{2} z_{2}^{2}
$$

The time derivative of $V_{2}$ along the solutions of (60) is

$$
\begin{aligned}
\dot{V}_{2} \leq & \dot{V}_{1}+z_{2}\left[z_{3}+\pi_{2}-k_{2} v_{0,1}-\frac{\partial \pi_{1}}{\partial y}\left(\xi_{2}\right.\right. \\
& \left.+f_{1,0}(y)+\omega^{T} \vartheta\right)-\frac{\partial \pi_{1}}{\partial \xi}\left(A_{0} \xi+k y\right)
\end{aligned}
$$

$$
\begin{aligned}
& -\frac{\partial \pi_{1}}{\partial v_{0}} \dot{v}_{0}-\frac{\partial \pi_{1}}{\partial \Xi}\left(A_{0} \Xi+\Phi^{T}(y)\right) \\
& -\frac{\partial \pi_{1}}{\partial \hat{\vartheta}} \Gamma\left(\tau_{1}-\mu \hat{\vartheta}\right)-\frac{\partial \pi_{1}}{\partial \hat{\beta}} \gamma_{1}\left(\sigma_{1}-\mu \hat{\beta}\right) \\
& -\frac{\partial \pi_{1}}{\partial \hat{p}} \gamma_{2}\left(\bar{\lambda}_{1}-\mu \hat{p}\right) \\
& -\frac{\partial \pi_{1}}{\partial \hat{\vartheta}}\left(\dot{\hat{\vartheta}}-\Gamma \tau_{1}+\Gamma \mu \hat{\vartheta}\right) \\
& -\frac{\partial \pi_{1}}{\partial \hat{\beta}}\left(\dot{\hat{\beta}}-\gamma_{1} \sigma_{1}+\gamma_{1} \mu \hat{\beta}\right) \\
& \left.-\frac{\partial \pi_{1}}{\partial \hat{p}}\left(\dot{\hat{p}}-\gamma_{2} \bar{\lambda}_{1}+\gamma_{2} \mu \hat{p}\right)-\frac{\partial \pi_{1}}{\partial \kappa} \dot{\kappa}\right] \\
& +\left|\frac{\partial \pi_{1}}{\partial y} p^{*} e_{2} z_{2}\right|+\left|\frac{\partial \pi_{1}}{\partial y} \Delta_{1} z_{2}\right| \\
& +\left|\frac{\partial \pi_{1}}{\partial y} \varepsilon_{1}(y) z_{2}\right| \cdot
\end{aligned}
$$

By Assumption 1 and Young's inequality, using the similar derivations in Step 1, one obtains the following inequalities:

$$
\begin{aligned}
& \left|\frac{\partial \pi_{1}}{\partial y} p^{*} e_{2} z_{2}\right| \leq e^{T} e+p\left(\frac{\partial \pi_{1}}{\partial y}\right)^{2} z_{2}^{2} \\
\left|\frac{\partial \pi_{1}}{\partial y} \Delta_{1} z_{2}\right| & \left|\frac{\partial \pi_{1}}{\partial y}\left(p_{1}^{*} \psi_{11}(|y|)+p_{1}^{*} \psi_{12}(|\zeta|)\right) z_{2}\right| \\
\leq & p_{1}^{*}\left|\frac{\partial \pi_{1}}{\partial y} z_{2}\right| \psi_{11}(|y|) \\
& +\frac{1}{4} p\left(\frac{\partial \pi_{1}}{\partial y}\right)^{2} z_{2}^{2}+\psi_{12}^{2}(|\zeta|) \\
= & p_{1}^{*}\left|\frac{\partial \pi_{1}}{\partial y} z_{2}\right|\left(\psi_{11}(|y|)-\psi_{11}(0)\right) \\
& +p_{1}^{*}\left|\frac{\partial \pi_{1}}{\partial y} z_{2}\right| \psi_{11}(0) \\
& +\frac{1}{4} p\left(\frac{\partial \pi_{1}}{\partial y}\right)^{2} z_{2}^{2}+\psi_{12}^{2}(|\zeta|) \\
\leq & p_{1}^{*}\left|\frac{\partial \pi_{1}}{\partial y} z_{2}\right||y| \bar{\psi}_{11}(|y|)+\frac{1}{2} p\left(\frac{\partial \pi_{1}}{\partial y}\right)^{2} z_{2}^{2} \\
+ & \psi_{12}^{2}(|\zeta|)+\psi_{11}^{2}(0), \\
& \leq p_{1}^{*}\left|\frac{\partial \pi_{1}}{\partial y} z_{2}\right||y| \bar{\psi}_{11}(|y|) \\
& \leq p_{1}^{*}\left|\frac{\partial \pi_{1}}{\partial y} z_{2}\right|\left(y \hat{\psi}_{11}(y)+d_{11}\right) \\
& +\frac{1}{2} p\left(\frac{\partial \pi_{1}}{\partial y}\right)^{2} z_{2}^{2} \hat{\psi}_{11}^{2}(y)+\frac{1}{4} y_{2}^{2}+\frac{1}{2} d_{11}^{2}, \\
\leq &
\end{aligned}
$$

where $d_{11}>0$ is a known constant, $\hat{\psi}_{11}$ is a known 
smooth function with $\hat{\psi}_{11}(0)=0$.

Substituting (65) into (64) yields

$$
\begin{aligned}
\left|\frac{\partial \pi_{1}}{\partial y} \Delta_{1} z_{2}\right| & \leq p\left(\frac{\partial \pi_{1}}{\partial y}\right)^{2} z_{2}^{2}\left(\hat{\psi}_{11}^{2}(y)+1\right) \\
& +\frac{1}{4} y^{2}+\psi_{12}^{2}(|\zeta|)+\psi_{11}^{2}(0)+\frac{1}{2} d_{11}^{2} .
\end{aligned}
$$

Note that

$$
\begin{aligned}
\left|\frac{\partial \pi_{1}}{\partial y} \varepsilon_{1}(y) z_{2}\right|-z_{2} \eta_{2} \beta \tanh & \left(\frac{z_{2} \eta_{2}}{\varsigma}\right) \\
& \leq 0.2785 \varsigma \beta=\varsigma^{\prime}
\end{aligned}
$$

where $\eta_{2}=-\partial \pi_{1} / \partial y$.

Substituting (63), (66) and (67) into (62), we obtain

$$
\begin{aligned}
\dot{V}_{2} \leq & -\left[\lambda_{\min }(Q)-5\right]\|e\|^{2}-z_{1} y \rho\left(y^{2}\right) \\
& +\frac{1}{4} y^{2}+z_{2}\left[z_{3}+\pi_{2}+\hat{b}_{0} z_{1}-k_{2} v_{0,1}\right. \\
& \left.-\frac{\partial \pi_{1}}{\partial y}\left(\xi_{2}+f_{1,0}(y)+\omega^{T} \hat{\vartheta}\right)+H_{2}\right] \\
& -z_{2} \frac{\partial \pi_{1}}{\partial \hat{\vartheta}}\left(\dot{\hat{\vartheta}}-\Gamma \tau_{1}+\Gamma \mu \hat{\vartheta}\right) \\
& -z_{2} \frac{\partial \pi_{1}}{\partial \hat{\beta}}\left(\dot{\hat{\beta}}-\gamma_{1} \sigma_{1}+\gamma_{1} \mu \hat{\beta}\right) \\
& -z_{2} \frac{\partial \pi_{1}}{\partial \hat{p}}\left(\dot{\hat{p}}-\gamma_{2} \bar{\lambda}_{1}+\gamma_{2} \mu \hat{p}\right) \\
& +\tilde{\vartheta}^{T}\left(\tau_{1}-\frac{\partial \pi_{1}}{\partial y} \omega z_{2}+\ell z_{2}-\Gamma^{-1} \dot{\hat{\vartheta}}\right) \\
& +\tilde{\beta}\left(\sigma_{1}+\eta_{2} z_{2} \tanh \left(\frac{z_{2} \eta_{2}}{\varsigma}\right)-\gamma_{1}^{-1} \dot{\hat{\beta}}\right) \\
& +\tilde{p}\left(\bar{\lambda}_{1}+\left(\frac{\partial \pi_{1}}{\partial y}\right)^{2} z_{2}^{2}\left(\hat{\psi}_{11}^{2}(y)+2\right)-\gamma_{2}^{-1} \dot{\hat{p}}\right) \\
& +\mu \tilde{\kappa} \hat{\kappa}+\|P\|^{2}\left(\sum_{k=1}^{n} \psi_{k 2}(|\zeta|)\right)^{2} \\
& +2 \psi_{12}^{2}(|\zeta|)+\|P\|^{2} \beta^{2}+\|P\|^{2} d_{\psi}^{0}+2 \varsigma^{\prime} \\
& +2 \psi_{11}^{2}(0)+\frac{3}{2} d_{11}^{2},
\end{aligned}
$$

where

$$
\begin{aligned}
& H_{2}=-\frac{\partial \pi_{1}}{\partial \xi}\left(A_{0} \xi+k y\right)-\frac{\partial \pi_{1}}{\partial \Xi}\left(A_{0} \Xi+\Phi^{T}(y)\right) \\
&-\frac{\partial \pi_{1}}{\partial v_{0}} \dot{v}_{0}-\frac{\partial \pi_{1}}{\partial \kappa} \dot{\kappa}-\frac{\partial \pi_{1}}{\partial \hat{\vartheta}} \Gamma\left(\tau_{1}-\mu \hat{\vartheta}\right) \\
&-\frac{\partial \pi_{1}}{\partial \hat{\beta}} \gamma_{1}\left(\sigma_{1}-\mu \hat{\beta}\right)-\frac{\partial \pi_{1}}{\partial \hat{p}} \gamma_{2}\left(\bar{\lambda}_{1}-\mu \hat{p}\right) \\
&-\hat{\beta} \eta_{2} \tanh \left(\frac{z_{2} \eta_{2}}{\varsigma}\right)+\hat{p}\left(\frac{\partial \pi_{1}}{\partial y}\right)^{2} z_{2}^{2}\left(\hat{\psi}_{11}^{2}(y)+2\right), \\
& \ell=\left[\begin{array}{llll}
z_{1} & 0 & \ldots & 0
\end{array}\right]^{T} .
\end{aligned}
$$

Choose the tuning functions and parameters adaptation laws as follows:

$$
\begin{aligned}
\tau_{2} & =\tau_{1}-z_{2}\left(\frac{\partial \pi_{1}}{\partial y} \omega-\ell\right), \\
\tau_{i} & =\tau_{i-1}-z_{i} \frac{\partial \pi_{i-1}}{\partial y} \omega, \quad i=3, \ldots, n, \\
\sigma_{i} & =\sigma_{i-1}+z_{i} \eta_{i} \tanh \left(\frac{z_{i} \eta_{i}}{\varsigma}\right), \quad i=2, \ldots, n, \\
\bar{\lambda}_{i} & =\bar{\lambda}_{i-1}+\left(\frac{\partial \pi_{i-1}}{\partial y}\right)^{2} z_{i}^{2}\left(\hat{\psi}_{11}^{2}(y)+2\right), \quad i=2, \ldots, n, \\
\dot{\hat{\vartheta}} & =\Gamma\left(\tau_{n}-\mu \hat{\vartheta}\right), \\
\dot{\hat{\beta}} & =\gamma_{1}\left(\sigma_{n}-\mu \hat{\beta}\right), \\
\dot{\hat{p}} & =\gamma_{2}\left(\bar{\lambda}_{n}-\mu \hat{p}\right),
\end{aligned}
$$

where

$$
\eta_{i}=-\frac{\partial \pi_{i-1}}{\partial y}, \quad i=2, \ldots, n
$$

Define

$$
\begin{aligned}
-\frac{\partial \pi_{1}}{\partial \hat{\vartheta}}\left(\dot{\hat{\vartheta}}-\Gamma \tau_{1}+\Gamma \mu \hat{\vartheta}\right) & =\sum_{j=2}^{n} \Delta_{1, j} z_{j}, \\
-\frac{\partial \pi_{1}}{\partial \hat{\beta}}\left(\dot{\hat{\beta}}-\gamma_{1} \sigma_{1}+\gamma_{1} \mu \hat{\beta}\right) & =\sum_{j=2}^{n} \Lambda_{1, j} z_{j}, \\
-\frac{\partial \pi_{1}}{\partial \hat{p}}\left(\dot{\hat{p}}-\gamma_{2} \bar{\lambda}_{1}+\gamma_{2} \mu \hat{p}\right) & =\sum_{j=2}^{n} \mathrm{~A}_{1, j} z_{j},
\end{aligned}
$$

where

$$
\begin{aligned}
\Delta_{1, j} & =\frac{\partial \pi_{1}}{\partial \hat{\vartheta}} \Gamma \frac{\partial \pi_{j-1}}{\partial y} \omega \\
\Lambda_{1, j} & =\frac{\partial \pi_{1}}{\partial \hat{\beta}} \gamma_{1} \eta_{j} \tanh \left(\frac{\eta_{j} z_{j}}{\varsigma}\right), \\
\mathrm{A}_{1, j} & =\frac{\partial \pi_{1}}{\partial \hat{p}} \gamma_{2}\left(\frac{\partial \pi_{j-1}}{\partial y}\right)^{2} z_{j}^{2}\left(\hat{\psi}_{11}^{2}(y)+2\right),
\end{aligned}
$$

$i=2, \ldots, n$.

Choose the stabilizing control function $\pi_{2}$ as

$$
\begin{aligned}
\pi_{2}= & -\hat{b}_{0} z_{1}-c_{2} z_{2}+\frac{\partial \pi_{1}}{\partial y}\left(\xi_{2}\right. \\
& \left.+f_{1,0}(y)+\omega^{T} \hat{\vartheta}\right)+k_{2} v_{0,1} \\
& -\left(\Delta_{1,2}+\Lambda_{1,2}+\mathrm{A}_{1,2}\right)-H_{2},
\end{aligned}
$$

where $c_{2}>0$ is a design constant. 
Substituting (69) and (71)-(79) into (68) yields

$$
\begin{aligned}
\dot{V}_{2} \leq & -\left[\lambda_{\min }(Q)-5\right]\|e\|^{2}-z_{1} y \rho\left(y^{2}\right) \\
& +\frac{1}{4} y^{2}+z_{2} z_{3}+\tilde{\vartheta}^{T}\left(\tau_{2}-\Gamma^{-1} \dot{\hat{\vartheta}}\right)+\mu \tilde{\kappa} \hat{\kappa} \\
& +\tilde{\beta}\left(\sigma_{2}-\gamma_{1}^{-1} \dot{\hat{\beta}}\right)+\tilde{p}\left(\bar{\lambda}_{2}-\gamma_{2}^{-1} \dot{\hat{p}}\right) \\
& +\|P\|^{2}\left(\sum_{k=1}^{n} \psi_{k 2}(|\zeta|)\right)^{2}+2 \psi_{12}^{2}(|\zeta|) \\
& +\|P\|^{2} \beta^{2}+\sum_{j=3}^{n}\left(\Delta_{1, j}+\Lambda_{1, j}+\mathrm{A}_{1, j}\right) z_{2} z_{j} \\
& +\|P\|^{2} d_{\psi}^{0}+2 \varsigma^{\prime}+2 \psi_{11}^{2}(0)+\frac{3}{2} d_{11}^{2} .
\end{aligned}
$$

Step $i(i=3, \ldots, n-1)$ : A similar procedure in Step 2 is employed recursively for consemtive steps. The time derivative of $z_{i}$ along (36) is

$$
\begin{aligned}
\dot{z}_{i}= & v_{0, i+1}-k_{i} v_{0,1}-\frac{\partial \pi_{i-1}}{\partial y}\left(\xi_{2}+f_{1,0}(y)\right. \\
& \left.+\omega^{T} \vartheta+p^{*} e_{2}+\Delta_{1}+\varepsilon_{1}(y)\right) \\
& -\frac{\partial \pi_{i-1}}{\partial \xi}\left(A_{0} \xi+k y\right) \\
& -\frac{\partial \pi_{i-1}}{\partial \Xi}\left(A_{0} \Xi+\Phi^{T}(y)\right)-\frac{\partial \pi_{i-1}}{\partial v_{0}} \dot{v}_{0} \\
& -\frac{\partial \pi_{i-1}}{\partial \kappa} \dot{\kappa}-\frac{\partial \pi_{i-1}}{\partial \hat{\vartheta}} \Gamma\left(\tau_{i-1}-\mu \hat{\vartheta}\right) \\
& -\frac{\partial \pi_{i-1}}{\partial \hat{\beta}} \gamma_{1}\left(\sigma_{i-1}-\mu \hat{\beta}\right) \\
& -\frac{\partial \pi_{i-1}}{\partial \hat{p}} \gamma_{2}\left(\bar{\lambda}_{i-1}-\mu \hat{p}\right) \\
& -\frac{\partial \pi_{i-1}}{\partial \hat{\vartheta}}\left(\dot{\hat{\vartheta}}-\Gamma \tau_{i-1}+\Gamma \mu \hat{\vartheta}\right) \\
& -\frac{\partial \pi_{i-1}}{\partial \hat{\beta}}\left(\dot{\hat{\beta}}-\gamma_{1} \sigma_{i-1}+\gamma_{1} \mu \hat{\beta}\right) \\
& -\frac{\partial \pi_{i-1}}{\partial \hat{p}}\left(\dot{\hat{p}}-\gamma_{2} \bar{\lambda}_{i-1}+\gamma_{2} \mu \hat{p}\right) .
\end{aligned}
$$

Consider the following Lyapunov function:

$$
V_{i}=V_{i-1}+\frac{1}{2} z_{i}^{2}
$$

The time derivative of $V_{i}$ along the solutions of (81) is

$$
\begin{aligned}
\dot{V}_{i} \leq & \dot{V}_{i-1}+z_{i}\left[z_{i+1}+\pi_{i}-k_{i} v_{0,1}\right. \\
& -\frac{\partial \pi_{i-1}}{\partial y}\left(\xi_{2}+f_{1,0}(y)+\omega^{T} \vartheta\right) \\
& -\frac{\partial \pi_{i-1}}{\partial \xi}\left(A_{0} \xi+k y\right) \\
& -\frac{\partial \pi_{i-1}}{\partial \Xi}\left(A_{0} \Xi+\Phi^{T}(y)\right)-\frac{\partial \pi_{i-1}}{\partial v_{0}} \dot{v}_{0}
\end{aligned}
$$

$$
\begin{aligned}
& -\frac{\partial \pi_{i-1}}{\partial \kappa} \dot{\kappa}-\frac{\partial \pi_{i-1}}{\partial \hat{\vartheta}} \Gamma\left(\tau_{i-1}-\mu \hat{\vartheta}\right) \\
& -\frac{\partial \pi_{i-1}}{\partial \hat{\beta}} \gamma_{1}\left(\sigma_{i-1}-\mu \hat{\beta}\right) \\
& -\frac{\partial \pi_{i-1}}{\partial \hat{p}} \gamma_{2}\left(\bar{\lambda}_{i-1}-\mu \hat{p}\right) \\
& -\frac{\partial \pi_{i-1}}{\partial \hat{\vartheta}}\left(\dot{\hat{\vartheta}}-\Gamma \tau_{i-1}+\Gamma \mu \hat{\vartheta}\right) \\
& -\frac{\partial \pi_{i-1}}{\partial \hat{\beta}}\left(\dot{\hat{\beta}}-\gamma_{1} \sigma_{i-1}+\gamma_{1} \mu \hat{\beta}\right) \\
& \left.-\frac{\partial \pi_{i-1}}{\partial \hat{p}}\left(\dot{\hat{p}}-\gamma_{2} \bar{\lambda}_{i-1}+\gamma_{2} \mu \hat{p}\right)\right] \\
& +\left|\frac{\partial \pi_{i-1}}{\partial y} p^{*} e_{2} z_{i}\right|+\left|\frac{\partial \pi_{i-1}}{\partial y} \Delta_{1} z_{i}\right| \\
& +\left|\frac{\partial \pi_{i-1}}{\partial y} \varepsilon_{1}(y) z_{i}\right| .
\end{aligned}
$$

By Young's inequality and Assumption 1, one obtains the following inequalities:

$$
\begin{aligned}
& \left|\frac{\partial \pi_{i-1}}{\partial y} p^{*} e_{2} z_{i}\right| \leq e^{T} e+p\left(\frac{\partial \pi_{i-1}}{\partial y}\right)^{2} z_{i}^{2}, \\
& \left|\frac{\partial \pi_{i-1}}{\partial y} \Delta_{1} z_{i}\right| \\
& \leq\left|\frac{\partial \pi_{i-1}}{\partial y}\left(p_{1}^{*} \psi_{11}(|y|)+p_{1}^{*} \psi_{12}(|\zeta|)\right) z_{i}\right| \\
& \leq p_{1}^{*}\left|\frac{\partial \pi_{i-1}}{\partial y} z_{i}\right| \psi_{11}(|y|) \\
& +\frac{1}{4} p\left(\frac{\partial \pi_{i-1}}{\partial y}\right)^{2} z_{i}^{2}+\psi_{12}^{2}(|\zeta|) \\
& \leq p_{1}^{*}\left|\frac{\partial \pi_{i-1}}{\partial y} z_{i}\right||y| \bar{\psi}_{11}(|y|) \\
& +\frac{1}{2} p\left(\frac{\partial \pi_{i-1}}{\partial y}\right)^{2} z_{i}^{2}+\psi_{12}^{2}(|\zeta|)+\psi_{11}^{2}(0) \\
& p_{1}^{*}\left|\frac{\partial \pi_{i-1}}{\partial y} z_{i}\right||y| \bar{\psi}_{11}(|y|) \\
& \leq p_{1}^{*}\left|\frac{\partial \pi_{i-1}}{\partial y} z_{i}\right|\left(y \hat{\psi}_{11}(y)+d_{11}\right) \\
& \leq p\left(\frac{\partial \pi_{i-1}}{\partial y}\right)^{2} z_{i}^{2} \hat{\psi}_{11}^{2}(y) \\
& +\frac{1}{4} y^{2}+\frac{1}{2} p\left(\frac{\partial \pi_{i-1}}{\partial y}\right)^{2} z_{i}^{2}+\frac{1}{2} d_{11}^{2} \\
& \left|\frac{\partial \pi_{i-1}}{\partial y} \Delta_{1} z_{i}\right| \\
& \leq p\left(\frac{\partial \pi_{i-1}}{\partial y}\right)^{2} z_{i}^{2}\left(\hat{\psi}_{11}^{2}(y)+1\right) \\
& +\frac{1}{4} y^{2}+\psi_{12}^{2}(|\zeta|)+\psi_{11}^{2}(0)+\frac{1}{2} d_{11}^{2}
\end{aligned}
$$




$$
\begin{aligned}
& \left|\frac{\partial \pi_{i-1}}{\partial y} \varepsilon_{1}(y) z_{i}\right|-z_{i} \eta_{i} \beta \tanh \left(\frac{z_{i} \eta_{i}}{\varsigma}\right) \\
& \quad \leq 0.2785 \varsigma \beta=\varsigma^{\prime},
\end{aligned}
$$

where $\eta_{i}=-\partial \pi_{i-1} / \partial y$.

Substituting (84), (87) and (88) into (83) gives

$$
\begin{aligned}
& \dot{V}_{i} \leq-\left[\lambda_{\min }(Q)-(i+3)\right]\|e\|^{2}-z_{1} y \rho\left(y^{2}\right) \\
& +\frac{i-1}{4} y^{2}+z_{i}\left[z_{i+1}+\pi_{i}-k_{i} v_{0,1}\right. \\
& \left.-\frac{\partial \pi_{i-1}}{\partial y}\left(\xi_{2}+f_{1,0}(y)+\omega^{T} \hat{\vartheta}\right)+H_{i}\right] \\
& -z_{i} \frac{\partial \pi_{i-1}}{\partial \hat{\vartheta}}\left(\dot{\hat{\vartheta}}-\Gamma \tau_{i-1}+\Gamma \mu \hat{\vartheta}\right) \\
& -z_{i} \frac{\partial \pi_{i-1}}{\partial \hat{\beta}}\left(\dot{\hat{\beta}}-\gamma_{1} \sigma_{i-1}+\gamma_{1} \mu \hat{\beta}\right) \\
& -z_{i} \frac{\partial \pi_{i-1}}{\partial \hat{p}}\left(\dot{\hat{p}}-\gamma_{2} \bar{\lambda}_{i-1}+\gamma_{2} \mu \hat{p}\right) \\
& +\tilde{\vartheta}^{T}\left(\tau_{i-1}-\frac{\partial \pi_{i-1}}{\partial y} \omega z_{i}-\Gamma^{-1} \dot{\hat{\vartheta}}\right) \\
& +\tilde{\beta}\left(\sigma_{i-1}+\eta_{i} z_{i} \tanh \left(\frac{z_{i} \eta_{i}}{\varsigma}\right)-\gamma_{1}^{-1} \dot{\hat{\beta}}\right) \\
& +\tilde{p}\left(\bar{\lambda}_{i-1}+\left(\frac{\partial \pi_{i-1}}{\partial y}\right)^{2} z_{i}^{2}\left(\hat{\psi}_{11}^{2}(y)+2\right)\right. \\
& \left.-\gamma_{2}^{-1} \dot{\hat{p}}\right)+\mu \tilde{\kappa} \hat{\kappa}-\sum_{j=1}^{i-1} c_{j} z_{j}^{2} \\
& +\|P\|^{2}\left(\sum_{k=1}^{n} \psi_{k 2}(|\zeta|)\right)^{2}+i \psi_{12}^{2}(|\zeta|) \\
& +\|P\|^{2} \beta^{2}+\|P\|^{2} d_{\psi}^{0}+i \varsigma^{\prime} \\
& +i \psi_{11}^{2}(0)+\frac{i+1}{2} d_{11}^{2} \text {, } \\
& H_{i}=-\frac{\partial \pi_{i-1}}{\partial \xi}\left(A_{0} \xi+k y\right)-\frac{\partial \pi_{i-1}}{\partial \Xi}\left(A_{0} \Xi+\Phi^{T}(y)\right) \\
& -\frac{\partial \pi_{i-1}}{\partial v_{0}} \dot{v}_{0}-\frac{\partial \pi_{i-1}}{\partial \kappa} \dot{\kappa}-\frac{\partial \pi_{i-1}}{\partial \hat{\vartheta}} \Gamma\left(\tau_{i-1}-\mu \hat{\vartheta}\right) \\
& -\frac{\partial \pi_{i-1}}{\partial \hat{\beta}} \gamma_{1}\left(\sigma_{i-1}-\mu \hat{\beta}\right)-\frac{\partial \pi_{i-1}}{\partial \hat{p}} \gamma_{2}\left(\bar{\lambda}_{i-1}-\mu \hat{p}\right) \\
& -\hat{\beta} \eta_{i} \tanh \left(\frac{z_{i} \eta_{i}}{\varsigma}\right)+\hat{p}\left(\frac{\partial \pi_{i-1}}{\partial y}\right)^{2} z_{i}^{2}\left(\hat{\psi}_{11}^{2}(y)+2\right) .
\end{aligned}
$$

Define

$$
\begin{array}{r}
-\frac{\partial \pi_{i-1}}{\partial \hat{\vartheta}}\left(\dot{\hat{\vartheta}}-\Gamma \tau_{i-1}+\Gamma \mu \hat{\vartheta}\right)=\sum_{j=i}^{n} \Delta_{i, j} z_{j}, \\
-\frac{\partial \pi_{i-1}}{\partial \hat{\beta}}\left(\dot{\hat{\beta}}-\gamma_{1} \sigma_{i-1}+\gamma_{1} \mu \hat{\beta}\right)=\sum_{j=i}^{n} \Lambda_{i, j} z_{j}, \\
-\frac{\partial \pi_{i-1}}{\partial \hat{p}}\left(\dot{\hat{p}}-\gamma_{2} \bar{\lambda}_{i-1}+\gamma \mu \hat{p}\right)=\sum_{j=i}^{n} \mathrm{~A}_{i, j} z_{j},
\end{array}
$$

where

$$
\begin{aligned}
\Delta_{i, j} & =\sum_{j=i}^{n} \frac{\partial \pi_{i-1}}{\partial \hat{\vartheta}} \Gamma \frac{\partial \pi_{j-1}}{\partial y} \omega \\
\Lambda_{i, j} & =\sum_{j=i}^{n} \frac{\partial \pi_{i-1}}{\partial \hat{\beta}} \gamma \eta_{j} \tanh \left(\frac{\eta_{j} z_{j}}{\varsigma}\right), \\
\mathrm{A}_{i, j} & =\sum_{j=i}^{n} \frac{\partial \pi_{i-1}}{\partial \hat{\vartheta}} \gamma_{2}\left(\frac{\partial \pi_{j-1}}{\partial y}\right)^{2} z_{j}^{2}\left(\hat{\psi}_{11}^{2}(y)+2\right) .
\end{aligned}
$$

Choose the stabilizing control function $\pi_{i}$ as

$$
\begin{aligned}
\pi_{i}= & -z_{i-1}-c_{i} z_{i}+k_{i} v_{0,1} \\
& +\frac{\partial \pi_{i-1}}{\partial y}\left(\xi_{2}+f_{1,0}(y)+\omega^{T} \hat{\vartheta}\right) \\
& -\sum_{k=2}^{i-1}\left(\Delta_{k, i}+\Lambda_{k, i}+\mathrm{A}_{k, i}\right) z_{k}-H_{i},
\end{aligned}
$$

where $c_{i}>0$ is a design constant. Substituting (90)-(93) into (89) and repeating procedures in Step 2, we have

$$
\begin{aligned}
\dot{V}_{i} \leq & -\left[\lambda_{\min }(Q)-(i+3)\right]\|e\|^{2}-z_{1} y \rho\left(y^{2}\right) \\
& +\frac{i-1}{4} y^{2}-\sum_{j=1}^{i} c_{j} z_{j}^{2}+\tilde{\vartheta}^{T}\left(\tau_{i}-\Gamma^{-1} \dot{\hat{\vartheta}}\right) \\
& +\tilde{\beta}\left(\sigma_{i}-\gamma_{1}^{-1} \dot{\hat{\beta}}\right)+\tilde{p}\left(\bar{\lambda}_{i}-\gamma_{2}^{-1} \dot{\hat{p}}\right)+\mu \tilde{\kappa} \hat{\kappa} \\
& +\sum_{j=i+1}^{n} \sum_{k=2}^{i}\left(\Delta_{k-1, j}+\Lambda_{k-1, j}\right) z_{k} z_{j} \\
& +\|P\|^{2}\left(\sum_{k=1}^{n} \psi_{k 2}(|\zeta|)\right)^{2}+z_{i} z_{i+1} \\
& +i \psi_{12}^{2}(|\zeta|)+\|P\|^{2} \beta^{2}+\|P\|^{2} d_{\psi}^{0}+i \varsigma^{\prime} \\
& +i \psi_{11}^{2}(0)+\frac{i+1}{2} d_{11}^{2}
\end{aligned}
$$

Step $n$ : In the final design step, the actual control input $u$ appears. Consider the overall Lyapunov function as

$$
V_{n}=V_{n-1}+\frac{1}{2} z_{n}^{2}
$$

Using (33) and (34), the time derivative of $V_{n}$ is

$$
\begin{aligned}
\dot{V}_{n} \leq & -\left[\lambda_{\min }(Q)-(n+3)\right]\|e\|^{2}-z_{1} y \rho\left(y^{2}\right) \\
& +\frac{n-1}{4} y^{2}+z_{n}\left[\sigma(y) u-k_{n} v_{0,1}\right. \\
& \left.-\frac{\partial \pi_{n-1}}{\partial y}\left(\xi_{2}+f_{1,0}(y)+\omega^{T} \hat{\vartheta}\right)+H_{n}\right] \\
& -\frac{\partial \pi_{n-1}}{\partial \hat{\vartheta}}\left(\dot{\hat{\vartheta}}-\Gamma \tau_{n-1}+\Gamma \mu \hat{\vartheta}\right) \\
& -\frac{\partial \pi_{n-1}}{\partial \hat{\beta}}\left(\dot{\hat{\beta}}-\gamma_{1} \sigma_{n-1}+\gamma_{1} \mu \hat{\beta}\right)
\end{aligned}
$$




$$
\begin{aligned}
& -\frac{\partial \pi_{n-1}}{\partial \hat{p}}\left(\dot{\hat{p}}-\gamma_{2} \bar{\lambda}_{n-1}+\gamma_{2} \mu \hat{p}\right) \\
& +\tilde{\vartheta}^{T}\left(\tau_{n-1}-\frac{\partial \pi_{n-1}}{\partial y} \omega z_{n}-\Gamma^{-1} \dot{\hat{\vartheta}}\right) \\
& +\tilde{\beta}\left(\sigma_{n-1}+\eta_{n} z_{n} \tanh \left(\frac{z_{n} \eta_{n}}{\varsigma}\right)-\gamma_{1}^{-1} \dot{\hat{\beta}}\right) \\
& +\tilde{p}\left(\bar{\lambda}_{n-1}+\left(\frac{\partial \pi_{n-1}}{\partial y}\right)^{2} z_{n}^{2}\left(\hat{\psi}_{11}^{2}(y)+2\right)\right. \\
& \left.-\gamma_{2}^{-1} \dot{\hat{p}}\right)+\mu \tilde{\kappa} \hat{\kappa}-\sum_{j=1}^{n-1} c_{j} z_{j}^{2} \\
& +\|P\|^{2}\left(\sum_{k=1}^{n} \psi_{k 2}(|\zeta|)\right)^{2}+n \psi_{12}^{2}(|\zeta|) \\
& +\|P\|^{2} \beta^{2}+\|P\|^{2} d_{\psi}^{0}+n \varsigma^{\prime} \\
& +n \psi_{11}^{2}(0)+\frac{n+1}{2} d_{11}^{2},
\end{aligned}
$$

where

$$
\begin{aligned}
H_{n}= & -\frac{\partial \pi_{n-1}}{\partial \xi}\left(A_{0} \xi+k y\right)-\frac{\partial \pi_{n-1}}{\partial \Xi}\left(A_{0} \Xi+\Phi^{T}(y)\right) \\
& -\frac{\partial \pi_{n-1}}{\partial v_{0}} \dot{v}_{0}-\frac{\partial \pi_{n-1}}{\partial \kappa} \dot{\kappa}-\frac{\partial \pi_{n-1}}{\partial \hat{\vartheta}} \Gamma\left(\tau_{n-1}-\mu \hat{\vartheta}\right) \\
& -\frac{\partial \pi_{n-1}}{\partial \hat{\beta}} \gamma_{1}\left(\sigma_{n-1}-\mu \hat{\beta}\right) \\
& -\frac{\partial \pi_{n-1}}{\partial \hat{p}} \gamma_{2}\left(\bar{\lambda}_{n-1}-\mu \hat{p}\right) \\
& -\hat{\beta} \eta_{n} \tanh \left(\frac{z_{n} \eta_{n}}{\varsigma}\right)+\hat{p}\left(\frac{\partial \pi_{n-1}}{\partial y}\right)^{2} z_{n}^{2}\left(\hat{\psi}_{11}^{2}(y)+2\right) .
\end{aligned}
$$

By completing the squares,

$$
\begin{aligned}
\mu \tilde{\vartheta}^{T} \hat{\vartheta} & =\mu \tilde{\vartheta}^{T}(\vartheta-\tilde{\vartheta})=\mu \tilde{\vartheta}^{T} \vartheta-\mu\|\tilde{\vartheta}\|^{2} \\
& \leq-\frac{1}{2} \mu\|\tilde{\vartheta}\|^{2}+\frac{1}{2} \mu\|\vartheta\|^{2}, \\
\mu \tilde{\beta} \hat{\beta} & \leq-\frac{1}{2} \mu\|\tilde{\beta}\|^{2}+\frac{1}{2} \mu\|\beta\|^{2}, \\
\mu \tilde{p} \hat{p} & \leq-\frac{1}{2} \mu\|\tilde{p}\|^{2}+\frac{1}{2} \mu\|p\|^{2}, \\
\mu \tilde{\kappa} \hat{\kappa} & \leq-\frac{1}{2} \mu\|\tilde{\kappa}\|^{2}+\frac{1}{2} \mu\|\kappa\|^{2} .
\end{aligned}
$$

Substituting (99)-(102) into (98) results in

$$
\begin{aligned}
\dot{V}_{n} \leq & -\left[\lambda_{\min }(Q)-(n+3)\right]\|e\|^{2}-\sum_{j=1}^{n} c_{j} z_{j}^{2} \\
& -\frac{1}{2} \mu\left(\|\tilde{\vartheta}\|^{2}+\|\tilde{\beta}\|^{2}+\|\tilde{p}\|^{2}+\|\tilde{\kappa}\|^{2}\right) \\
& -y^{2}\left(\lambda^{\prime}\left(y^{2}\right) \rho\left(y^{2}\right)-\frac{n-1}{4}\right) \\
& +\|P\|^{2}\left(\sum_{k=1}^{n} \psi_{k 2}(|\zeta|)\right)^{2}+n \psi_{12}^{2}(|\zeta|)+D,
\end{aligned}
$$

where

$$
\begin{aligned}
D=\frac{1}{2} \mu\left(\|\vartheta\|^{2}+\|\beta\|^{2}+\|p\|^{2}\right)+\|P\|^{2} \beta^{2} \\
+\|P\|^{2} d_{\psi}^{0}+n \varsigma^{\prime}+n \psi_{11}^{2}(0)+\frac{n+1}{2} d_{11}^{2} .
\end{aligned}
$$

Assume that

$$
\lambda_{\min }(Q)-(n+3)>0 .
$$

In the sequel, we are to robustify the adaptive fuzzy

$$
\begin{aligned}
u= & \frac{1}{\sigma(y)}\left(k_{n} v_{0,1}+\frac{\partial \pi_{n-1}}{\partial y}\left(\xi_{2}+f_{1,0}(y)\right.\right. \\
& \left.+\omega^{T} \hat{\vartheta}\right)-c_{n} z_{n}-z_{n-1}-\sum_{k=2}^{n-1}\left(\Delta_{k-1, n}\right. \\
& \left.\left.+\Lambda_{k-1, n}+\mathrm{A}_{k-1, n}\right) z_{k}-H_{n}\right)
\end{aligned}
$$

where $c_{n}>0$ is a design constant.

Substituting (73)-(75) and (97) into (96) yields

$$
\begin{aligned}
\dot{V}_{n} \leq & -\left[\lambda_{\min }(Q)-(n+3)\right]\|e\|^{2}-z_{1} y \rho\left(y^{2}\right) \\
& +\frac{n-1}{4} y^{2}-\sum_{j=1}^{n} c_{j} z_{j}^{2}+\mu \tilde{\vartheta} \hat{\vartheta}+\mu \tilde{\beta} \hat{\beta} \\
& +\mu \tilde{p} \hat{p}+\mu \tilde{\kappa} \hat{\kappa}+\|P\|^{2}\left(\sum_{k=1}^{n} \psi_{k 2}(|\zeta|)\right)^{2} \\
& +n \psi_{12}^{2}(|\zeta|)+\|P\|^{2} \beta^{2}+\|P\|^{2} d_{\psi}^{0}+n \varsigma^{\prime} \\
& +n \psi_{11}^{2}(0)+\frac{n+1}{2} d_{11}^{2} .
\end{aligned}
$$
controller obtained in the preceding design procedures via the appropriate choice of design functions $\lambda\left(y^{2}\right)$ and $\rho\left(y^{2}\right)$ to check the conditions of small-gain Theorem 1 .

Firstly, choose a smooth function $\rho\left(y^{2}\right)$ as introduced in Step 1 to satisfy

$$
y^{2}\left[\lambda^{\prime}\left(y^{2}\right) \rho\left(y^{2}\right)-\frac{n-1}{4}\right] \geq c_{1} \lambda\left(y^{2}\right) .
$$

Because $\lambda^{\prime}\left(y^{2}\right) \neq 0$ for any $y$, as stated by Jiang (1999), such a smooth function always exists. Since each function $\psi_{i 2}$ is smooth and vanishes at the origin, there is a smooth class- $\kappa_{\infty}$ function $h$ such that

$$
\|P\|^{2}\left(\sum_{i=1}^{n} \psi_{i 2}(|\zeta|)\right)^{2}+n \psi_{12}^{2}(|\zeta|) \leq h\left(|\zeta|^{2}\right) .
$$

Let

$$
\begin{gathered}
c=\min \left\{\frac{\lambda_{\min }(Q)-(n+3)}{\lambda_{\max }(P)}, 2 c_{j}, \frac{\mu}{\lambda_{\max }\left(\Gamma^{-1}\right)},\right. \\
\left.\mu \gamma_{1}, \mu \gamma_{2}, \mu \gamma_{3} ; j=1, \ldots, n\right\} .
\end{gathered}
$$


Then (103) can be expressed as

$$
\dot{V}_{n} \leq-c V_{n}+h\left(|\zeta|^{2}\right)+D
$$

In order to use Lemma 1 and Theorem 1, one assumes that

$$
\begin{aligned}
\dot{V}_{n} & \leq-d_{1} V_{n}+d_{1} V_{n}-c V_{n}+h\left(|\zeta|^{2}\right)+D \\
& \leq-d_{1} V_{n}
\end{aligned}
$$

that is, for any $0<d_{1}<c$, (108) ensures that the following inequality holds:

$$
d_{1} V_{n}-c V_{n}+h\left(|\zeta|^{2}\right)+D \leq 0
$$

or, equivalently,

$$
V_{n} \geq \frac{h\left(|\zeta|^{2}\right)}{c-d_{1}}+\frac{D}{c-d_{1}} .
$$

From (3), one has

$$
\begin{gathered}
|\zeta| \leq \alpha_{1}^{-1}\left(V_{0}(\zeta)\right), \\
|\zeta| \geq \alpha_{2}^{-1}\left(V_{0}(\zeta)\right) .
\end{gathered}
$$

From (111) and (110), we obtain

$$
\frac{h\left(\alpha_{1}^{-1}\left(V_{0}(\zeta)\right)^{2}\right)}{c-d_{1}}+\frac{D}{c-d_{1}} \geq \frac{h\left(|\zeta|^{2}\right)}{c-d_{1}}+\frac{D}{c-d_{1}} .
$$

Therefore, (110) holds as long as the following inequality holds:

$$
V_{n} \geq \frac{h\left(\alpha_{1}^{-1}\left(V_{0}(\zeta)\right)^{2}\right)}{c-d_{1}}+\frac{D}{c-d_{1}} .
$$

On the other hand,

$$
\begin{aligned}
& \max \left\{\frac{2 h\left(\alpha_{1}^{-1}\left(V_{0}(\zeta)\right)^{2}\right)}{c-}, \frac{2 D}{c-d_{1}}\right\} \\
& \geq \frac{h\left(\alpha_{1}^{-1}\left(V_{0}(\zeta)\right)^{2}\right)}{c-d_{1}}+\frac{D}{c-d_{1}} .
\end{aligned}
$$

Therefore, if

$$
V_{n} \geq \max \left\{\frac{2 h\left(\alpha_{1}^{-1}\left(V_{0}(\zeta)\right)^{2}\right)}{c-d_{1}}, \frac{2 D}{c-d_{1}}\right\},
$$

it follows that (108) holds.

Secondly, in order to invoke Theorem 1 (the smallgain theorem), the function $\lambda\left(y^{2}\right)$ needs to be chosen appropriately such that for arbitrary $d_{2}>0$, the following inequality holds:

$$
\gamma^{-1} \circ \gamma_{0}(|y|) \leq \frac{1}{4} \lambda\left(y^{2}\right)+d_{2} \leq \frac{1}{2} V_{n}+d_{2},
$$

where the notation $\circ$ stands for the composition operator between two functions. Since $\gamma$ is a $\kappa_{\infty}$-function and $\gamma$ is an increasing function, we have

$$
\gamma\left(\frac{1}{2} V_{n}+d_{2}\right) \leq \gamma\left(V_{n}\right)+\gamma\left(2 d_{2}\right) .
$$

Substituting (116) and (117) into (3) results in

$$
\begin{aligned}
\frac{\partial V_{0}}{\partial \zeta} q(\zeta, y) & \leq-\alpha_{0}(|\zeta|)+\gamma\left(\gamma^{-1} \circ \gamma_{0}(|y|)\right)+d_{0} \\
& \leq-\alpha_{0}(|\zeta|)+\gamma\left(V_{n}\right)+\gamma\left(2 d_{2}\right)+d_{0} .
\end{aligned}
$$

For any given $0<d_{3}<1$, by (109) and (115), we obtain

$$
\begin{aligned}
\dot{V}_{0} \leq & -\alpha_{0}(|\zeta|)+\gamma\left(V_{n}\right)+\gamma\left(2 d_{2}\right)+d_{0} \\
\leq & -d_{3} \alpha_{0} \circ \alpha_{2}^{-1}\left(V_{0}(\zeta)\right) \\
& +d_{3} \alpha_{0} \circ \alpha_{2}^{-1}\left(V_{0}(\zeta)\right)-\alpha_{0} \circ \alpha_{2}^{-1}\left(V_{0}(\zeta)\right) \\
& +\gamma\left(V_{n}\right)+\gamma\left(2 d_{2}\right)+d_{0} .
\end{aligned}
$$

The following inequality holds:

$$
\dot{V}_{0} \leq-d_{3} \alpha_{0} \circ \alpha_{2}^{-1}\left(V_{0}(\zeta)\right)
$$

as long as

$$
\begin{aligned}
d_{3} \alpha_{0} \circ \alpha_{2}^{-1}\left(V_{0}(\zeta)\right) & -\alpha_{0} \circ \alpha_{2}^{-1}\left(V_{0}(\zeta)\right) \\
+ & \gamma\left(V_{n}\right)+\gamma\left(2 d_{2}\right)+d_{0} \leq 0
\end{aligned}
$$

or, equivalently,

$$
\alpha_{0} \circ \alpha_{2}^{-1}\left(V_{0}(\zeta)\right) \geq \frac{\gamma\left(V_{n}\right)}{1-d_{3}}+\frac{\gamma\left(2 d_{2}\right)+d_{0}}{1-d_{3}} .
$$

From (122), we get

$$
V_{0} \geq \alpha_{2} \circ \alpha_{0}^{-1}\left\{\frac{\gamma\left(V_{n}\right)}{1-d_{3}}+\frac{\gamma\left(2 d_{2}\right)+d_{0}}{1-d_{3}}\right\} \text {. }
$$

Since

$$
\begin{gathered}
\max \left\{\alpha_{2} \circ \alpha_{0}^{-1} \circ \frac{2 \gamma\left(V_{n}\right)}{1-d_{3}}, \alpha_{2} \circ \alpha_{0}^{-1} \circ \frac{2 \gamma\left(2 d_{2}\right)+2 d_{0}}{1-d_{3}}\right\} \\
\geq \alpha_{2} \circ \alpha_{0}^{-1}\left\{\frac{\gamma\left(V_{n}\right)}{1-d_{3}}+\frac{\gamma\left(2 d_{2}\right)+d_{0}}{1-d_{3}}\right\},
\end{gathered}
$$

for any given $d_{4}>0$, as long as

$$
\begin{aligned}
d_{4} V_{0} \geq \max \{ & d_{4} \alpha_{2} \circ \alpha_{0}^{-1} \circ \frac{2 \gamma\left(V_{n}\right)}{1-d_{3}}, \\
& \left.d_{4} \alpha_{2} \circ \alpha_{0}^{-1} \circ \frac{2 \gamma\left(2 d_{2}\right)+2 d_{0}}{1-d_{3}}\right\},
\end{aligned}
$$

it is sufficient to guarantee that the inequality (120) holds, that is, $\dot{V}_{0} \leq-d_{3} \alpha_{0} \circ \alpha_{2}^{-1}\left(V_{0}\right)$. From (108) and (115), the condition (8) is satisfied. The class $\kappa_{\infty}$-function for the $\left(x_{1}, v_{0}, z_{1}, \cdots, z_{n}, \hat{\beta}, \hat{p}, \hat{\vartheta}, \kappa\right)$ system with input $d_{4} V_{0}$ and output $V_{n}$ is given as

$$
\chi_{1}(s)=\frac{2 h\left(\alpha_{1}^{-1}\left(\frac{1}{d_{4}} s\right)^{2}\right)}{c-d_{1}} .
$$

Similarly, from (120) and (124), the condition (9) is also satisfied for the $z$-system with input $V_{n}$ and output $d_{4} V_{0}$. The gain function is

$$
\chi_{2}(s)=d_{4} \alpha_{2} \circ \alpha_{0}^{-1} \circ \frac{2 \gamma(s)}{1-d_{3}} .
$$


Finally, to check the conditions of Theorem 1 (Jiang et al., 1996), for any given $s>0$, we select any function $\gamma$ of class $\kappa_{\infty}$ such that

$$
\gamma(s)<\frac{1-d_{3}}{2} \alpha_{0} \circ \alpha_{2}^{-1} \circ \alpha_{1}\left(\sqrt{h^{-1}\left(\frac{c-d_{1}}{2} s\right)}\right) .
$$

From (125), (126) and (127), we obtain

$$
\chi_{1} \circ \chi_{2}(s)<s \text {. }
$$

Therefore, by (128) and Theorem 1, we conclude that the closed-loop system is ISpS, and the variables $x_{i}(t), e(t)$, $\vartheta, \beta, p, \kappa$ and $u(t)$ are uniformly ultimately bounded.

The above analysis and small-gain design are summarized in the following theorem.

Theorem 2. For the nonlinear system (1), under Assumptions 1-3, after the application of the above design procedures, the proposed adaptive fuzzy output feedback control scheme can guarantee that all the signals of the closed-loop system are uniformly ultimately bounded.

From the previous synthesis, we can obtain the following controller design procedure:

Step 1: Define the fuzzy IF-THEN rules and the membership functions, determine the fuzzy basis functions, and establish the fuzzy logic systems (11).

Step 2: Specify the observer gain vector $K$, such that $A_{0}$ is a strict Hurwitz matrix.

Step 3: Specify a positive definite matrix $Q=Q^{T}>0$, such that $\lambda_{\min }(Q)-(n+3)>0$, solving the matrix equation (19) to obtain positive definite matrix $P$.

Step 4: Select the appropriate design parameter $c_{1}>0$, by using (104), (116) and (127), construct functions $\rho\left(y^{2}\right)$ and $\lambda\left(y^{2}\right)$, then obtain the adaptation functions $\hat{\kappa}$ in (58) and the stabilizing function $\bar{\pi}_{1}$ in (54), i.e., the intermediate control function $\pi_{1}=\hat{\kappa} \bar{\pi}_{1}$.

Step 5: Select the appropriate design parameters $c_{i}>0$ $(i=2, \ldots, n), \Gamma=\Gamma^{T}>0, \gamma_{1}>0, \gamma_{2}>0, \gamma_{3}>0$, $\mu>0$. Compute the partial derivations of the stabilizing function $\pi_{i}$, for $i=1, \ldots, n-1$, then obtain the intermediate control function $\pi_{i}$ in (93). Finally, obtain $u$ in (97) and adaptation functions $\hat{\beta}, \hat{p}$ and $\hat{\vartheta}$, which are expressed by (73)-(75), respectively.

\section{Simulation}

In this section, a simulation example and simulation comparisons are presented to show the effectiveness of the proposed adaptive backstepping control scheme.
Example 1. Consider the second-order nonlinear system with unmodeled dynamics and dynamical disturbances

$$
\begin{aligned}
\dot{\zeta} & =-\zeta+0.125 y^{2}, \\
\dot{x}_{1} & =x_{2}+f_{1,0}(y)+f_{1}(y)+\Delta_{1}, \\
\dot{x}_{2} & =b_{0} u+f_{2,0}(y)+f_{2}(y)+\Delta_{2}, \\
y & =x_{1},
\end{aligned}
$$

where $f_{1,0}(y)=0$ and $f_{2,0}(y)=0$ are known functions. $f_{1}(y)=5 y^{2}$ and $f_{2}(y)=-0.5 y$ are unknown functions. $\dot{\zeta}=-\zeta+0.125 y^{2}$ stands for unmodeled dynamics, $\Delta_{1}=$ $\zeta^{2}$ and $\Delta_{2}=0.2 \zeta^{2}$ are nonlinear dynamical disturbances and $b_{0}=-4$.

Based on the following inequalities:

$$
\begin{aligned}
& \left|\Delta_{1}\right| \leq|\zeta|^{2}=0 .\left|x_{1}\right|^{2}+1 .|\zeta|^{2} \leq p_{1}^{*}\left|x_{1}\right|^{2}+p_{1}^{*}|\zeta|^{2}, \\
& \left|\Delta_{2}\right| \leq|\zeta|^{2}=0 .\left|x_{1}\right|^{2}+0.2 \cdot|\zeta|^{2} \leq p_{2}^{*}\left|x_{1}\right|^{2}+p_{2}^{*}|\zeta|^{2},
\end{aligned}
$$

set $\psi_{11}(s)=0, \psi_{21}(s)=0, \psi_{12}(s)=s^{2}, \psi_{22}(s)=s^{2}$. Then Assumption 1 is satisfied.

Taking $V_{0}=\zeta^{2}$, the time derivative of $V_{0}$ along (129) is

$$
\begin{aligned}
\dot{V}_{0} & =2 \zeta \dot{\zeta}=-2 \zeta^{2}+0.25 \zeta y^{2} \\
& \leq-1.875 \zeta^{2}+0.125 y^{4} \\
& \leq-\zeta^{2}+0.125 y^{4} .
\end{aligned}
$$

Defining $\alpha_{0}(s)=s^{2}, \gamma_{0}(s)=0.125 s^{4}, \alpha_{1}(s)=0.5 s^{2}$, $\alpha_{2}(s)=1.5 s^{2}$ and $d_{0}=0$, Assumption 2 holds.

According to the controller design procedures in the above section, the controller design is as follows:

Step 1: Since the functions $f_{1}(y)$ and $f_{1}(y)$ in (129) are zero at the point $y=0$, it usually takes a symmetrical interval about the origin $[-a, a]$. Then choose the fuzzy membership function to cover the interval $[-a, a]$ uniformly. In this way, the fuzzy logic system can achieve better approximating results. In this paper, we take $[-a, a]$ as $[-6,6]$ for the variable $y$. Therefore, define fuzzy membership functions as

$$
\begin{aligned}
& \mu_{F_{1}^{l}}(y)=\exp \left[-(y-6+2 l)^{2}\right], \quad l=1, \ldots, 5, \\
& \mu_{F_{2}^{l}}(y)=\exp \left[-(y-6+2 l)^{2}\right] \cdot \exp \left[-(y-12+l 4)^{2}\right], \\
& \quad l=1, \ldots, 5 .
\end{aligned}
$$

The fuzzy basis functions are expressed as

$$
\varphi_{1 j}(y)=\frac{\exp \left[-(y-6+2 j)^{2}\right]}{\sum_{n=1}^{5} \exp \left[-(y-6+2 n)^{2}\right]}, \quad l=1, \ldots, 5,
$$

$$
\begin{aligned}
& \varphi_{2 j}(y) \\
& =\frac{\exp \left[-(y-6+2 j)^{2}\right] \times \exp \left[-(y-12+4 j)^{2}\right]}{\sum_{n=1}^{5} \exp \left[-(y-6+2 n)^{2}\right] \times \exp \left[-(y-12+4 n)^{2}\right]}, \\
& \quad l=1, \ldots, 5
\end{aligned}
$$


Therefore, the unknown functions $f_{i}(y)(i=1,2)$ can be approximated as $f_{1}(y)=\theta_{1}^{* T} \varphi_{1}(y)+\varepsilon_{1}(y)$ and $f_{2}(y)=$ $\theta_{2}^{* T} \varphi_{2}(y)+\varepsilon_{2}(y)$

Step 2: Specify the observer gain vector

$$
K=\left[k_{1}, k_{2}\right]^{T}=[15,15]^{T},
$$

such that $A_{0}$ is a strict Hurwitz matrix.

Step 3: Given a positive definite matrix $Q=8 I$, such that $\lambda_{\min }(Q)-(n+3)>0$, by solving the Lyapunov equation (19), a positive definite matrix

$$
P=\left[\begin{array}{cc}
4.2667 & -4 \\
-4 & 4.2844
\end{array}\right]
$$

is obtained.

Step 4: Selecting the design parameter $c_{1}=2$ and design function $\lambda\left(y^{2}\right)=10 y^{8}+0.1 y^{2}$, we obtain the function

$$
\phi_{11}(y)=1+\frac{1}{2} \cdot \frac{1}{40 y^{6}+0.1} .
$$

Using (104), we choose function $\rho\left(y^{2}\right)=y^{2}+4.85$. Using (105) and (127), respectively, the design functions are $h(s)=280 s^{2}, \gamma(s)=0.007 s^{\frac{1}{2}}$ and $d_{1}=0.0001$. If we choose $d_{3}=0.0001, d_{2}=d_{4}=0.1$ and $c=1$, then the conditions of the small-gain-theorem are satisfied.

Define the parameter vector as

$$
\vartheta=\left[\begin{array}{lll}
b_{0} & \theta_{1}^{* T} & \theta_{2}^{* T}
\end{array}\right]^{T} .
$$

The filters are given as

$$
\begin{gathered}
\dot{\xi}=A_{0} \xi+\left[\begin{array}{l}
k_{1} \\
k_{2}
\end{array}\right] y, \\
\dot{\Xi}=A_{0} \Xi+\Phi, \quad \dot{v}_{0}=A_{0} v_{0}+\left[\begin{array}{l}
0 \\
1
\end{array}\right] u,
\end{gathered}
$$

where

$$
\begin{aligned}
\xi & =\left[\begin{array}{l}
\xi_{1} \\
\xi_{2}
\end{array}\right], & A_{0}=\left[\begin{array}{cc}
-k_{1} & 1 \\
-k_{2} & 0
\end{array}\right], \\
\Xi & =\left[\begin{array}{l}
\Xi_{(1)} \\
\Xi_{(2)}
\end{array}\right], & \Phi=\left[\begin{array}{cc}
\varphi_{1}^{T} & 0 \\
0 & \varphi_{2}^{T}
\end{array}\right], \\
v_{0}=\left[\begin{array}{l}
v_{01} \\
v_{02}
\end{array}\right] . & &
\end{aligned}
$$

The stabilization functions and the control laws are given by

$$
\begin{aligned}
\pi_{1}= & \hat{\kappa} \bar{\pi}_{1}, \\
\bar{\pi}_{1}= & -y \rho\left(y^{2}\right)-\xi_{2}-f_{1,0}(y)-\bar{\omega} \hat{\vartheta} \\
& -\frac{\|P\|^{2}}{\lambda^{\prime}} y \phi_{1}(y)-\hat{p} \phi_{11}(y) z_{1}-\eta_{1} \hat{\beta} \tanh \left(\frac{z_{1} \eta_{1}}{\varsigma}\right),
\end{aligned}
$$

where

$$
\begin{aligned}
& \omega=\left[v_{0,2}, \Phi_{(1)}^{T}(y)+\Xi_{(2)}\right]^{T}, \\
& \bar{\omega}=\left[0, \Phi_{(1)}^{T}(y)+\Xi_{(2)}\right]^{T} .
\end{aligned}
$$

Step 5: Controller $u$ and parameter adaptation laws are chosen as

$$
\begin{aligned}
u= & \frac{1}{\sigma(y)}\left(-\hat{b}_{0} z_{1}-c_{2} z_{2}+\frac{\partial \pi_{1}}{\partial y}\left(\xi_{2}+f_{1,0}(y)+\omega^{T} \hat{\vartheta}\right)\right. \\
& \left.+k_{2} v_{0,1}-\left(\Delta_{1,2}+\Lambda_{1,2}+\mathrm{A}_{1,2}\right)-H_{2}\right) \\
\dot{\hat{\vartheta}} & =\Gamma\left(\tau_{2}-\mu \hat{\vartheta}\right), \quad \dot{\hat{\beta}}=\gamma_{1}\left(\sigma_{2}-\mu \hat{\beta}\right), \\
\dot{\hat{p}} & =\gamma_{2}\left(\bar{\lambda}_{2}-\mu \hat{p}\right), \quad \dot{\hat{\kappa}}=-\gamma_{3}\left(\operatorname{sgn}\left(b_{0}\right) \bar{\pi}_{1} z_{1}+\mu \hat{\kappa}\right),
\end{aligned}
$$

where

$$
\begin{aligned}
\tau_{1} & =\bar{\omega} z_{1}, \\
\sigma_{1} & =z_{1} \eta_{1} \beta \tanh \left(\frac{z_{1} \eta_{1}}{\varsigma}\right), \\
\bar{\lambda}_{1} & =\phi_{11}(y) z_{1}^{2}, \\
\tau_{2} & =\bar{\omega} z_{1}+\ell z_{2}-z_{2} \frac{\partial \pi_{1}}{\partial y} \omega, \\
\sigma_{2} & =z_{1} \eta_{1} \tanh \left(\frac{z_{1} \eta_{1}}{\varsigma}\right)+z_{2} \eta_{2} \tanh \left(\frac{z_{2} \eta_{2}}{\varsigma}\right), \\
\lambda_{2} & =\phi_{11}(y) z_{1}^{2}+\left(\frac{\partial \pi_{1}}{\partial y}\right)^{2} z_{2}^{2}\left(\hat{\psi}_{11}^{2}(y)+2\right),
\end{aligned}
$$

$$
\begin{aligned}
H_{2}= & -\frac{\partial \pi_{1}}{\partial \xi}\left(A_{0} \xi+k y\right)-\frac{\partial \pi_{1}}{\partial \Xi}\left(A_{0} \Xi+\Phi^{T}(y)\right) \\
& -\frac{\partial \pi_{1}}{\partial v_{0}} \dot{v}_{0}-\frac{\partial \pi_{1}}{\partial \kappa} \dot{\kappa}-\frac{\partial \pi_{1}}{\partial \hat{\vartheta}} \Gamma\left(\tau_{1}-\mu \hat{\vartheta}\right) \\
& -\frac{\partial \pi_{1}}{\partial \hat{\beta}} \gamma_{1}\left(\sigma_{1}-\mu \hat{\beta}\right)-\frac{\partial \pi_{1}}{\partial \hat{p}} \gamma_{2}\left(\bar{\lambda}_{1}-\mu \hat{p}\right) \\
& -\hat{\beta} \eta_{2} \tanh \left(\frac{z_{2} \eta_{2}}{\varsigma}\right)+\hat{p}\left(\frac{\partial \pi_{1}}{\partial y}\right)^{2} z_{2}^{2}\left(\hat{\psi}_{11}^{2}(y)+2\right)
\end{aligned}
$$

The design parameters in the controller and adaptation laws are chosen as $c_{2}=0.5, \mu=0.9, \Gamma=1.2 I$, $\varsigma=0.1, \gamma_{1}=1.2, \gamma_{2}=1.2, \gamma_{3}=1.2$.

If the initial conditions are given as

$$
\begin{array}{rlrl}
x_{1}(0) & =0.12, & & \zeta(0)=0.1, \\
\hat{\kappa}(0) & =0.1, & \hat{p}(0)=1.5, \\
\hat{\vartheta}(0) & =[0.8,0,0,0,0,0,0,0,0,0,0]^{T}, & \\
\xi(0) & =\Xi(0)=v_{0}(0)=\hat{\beta}(0)=0 . &
\end{array}
$$

The simulation results are shown in Figs. 1-3, where Figs. 1 and 2 show the trajectories of $x_{1}, x_{2}, \hat{\hat{x}}_{1}$ and $\hat{\hat{x}}_{2}$, respectively, while Fig. 3. shows the trajectory of control input $u$. 


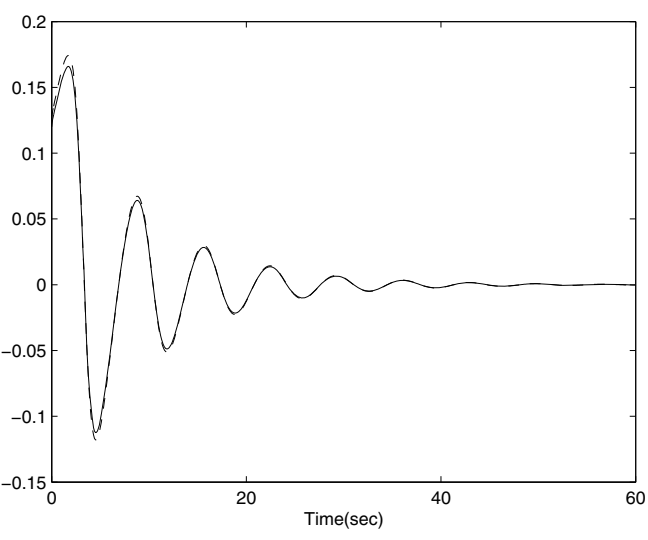

Fig. 1. Trajectories of $x_{1}$ (dashed) and $\hat{x}_{1}$ (solid).

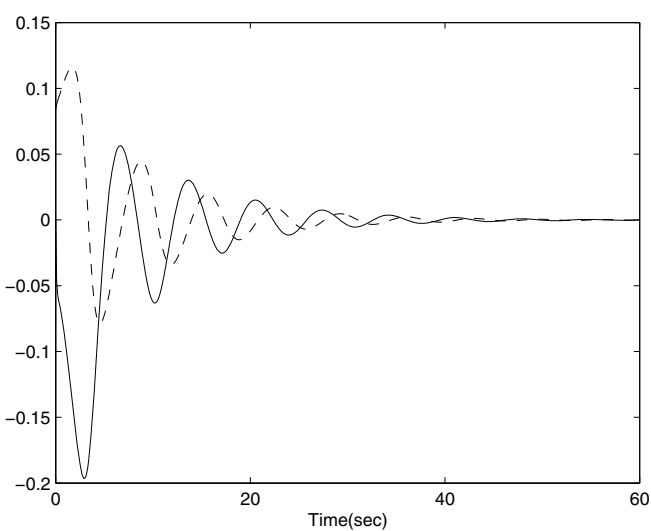

Fig. 2. Trajectories of $x_{2}$ (dashed) and $\hat{x}_{2}$ (solid).

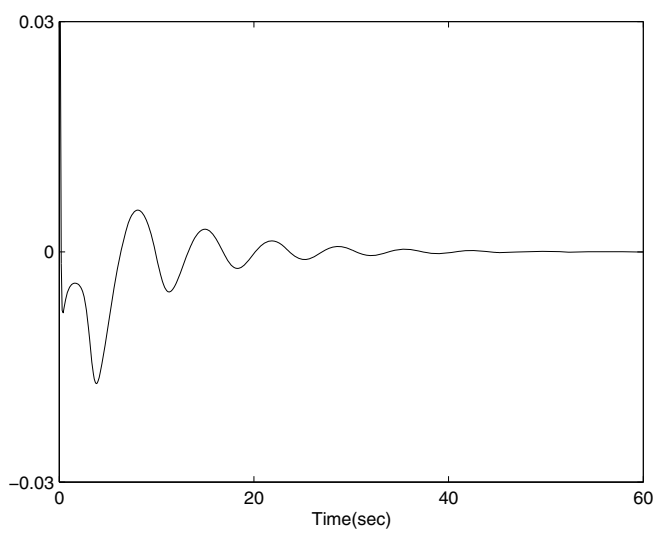

Fig. 3. System control input $u$.

Example 2. In order to further illustrate the effectiveness of the proposed control method, use the control scheme by Yang et al. (2005) to control the nonlinear system in Example 1.
Case 1: Consider the nonlinear system (129) without unmodeled dynamics and dynamical disturbances, and assume that $x_{1}$ and $x_{2}$ are both measured.

For this case, we use the same fuzzy membership functions and fuzzy logic systems as in Example 1 to approximate the unknown functions $f_{1}(y)$ and $f_{2}(y)$, respectively. According to Yang et al. (2005), the stabilization functions, the controller and adaptation laws are given by

$$
\begin{aligned}
\alpha_{1}= & -k_{1} z_{1}-\frac{1}{4 \gamma_{1}^{2}} z_{1} \lambda_{1} \xi_{1}^{T}\left(x_{1}\right) \xi_{1}\left(x_{1}\right) \\
& -\hat{\theta}_{1} \tanh \left(\frac{\hat{\theta}_{1} z_{1}}{\delta_{1}}\right), \\
\dot{\lambda}_{1}= & \Gamma_{11}\left[\frac{1}{4 \gamma_{1}^{2}} \lambda_{1} \xi_{1}^{T}\left(x_{1}\right) \xi_{1}\left(x_{1}\right) z_{1}^{2}-\sigma_{11}\left(\lambda_{1}-\lambda_{1}^{0}\right)\right], \\
\dot{\hat{\theta}}_{1}= & \Gamma_{12}\left[\left\|z_{1}\right\|-\sigma_{12}\left(\hat{\theta}_{1}-\theta_{1}^{0}\right)\right], \\
u= & -k_{2} z_{2}-z_{1}-\frac{1}{4 \gamma_{2}^{2}} z_{2} \lambda_{2} \xi_{2}^{T} \xi_{2}-\hat{\theta}_{2} z_{2} \tanh \left(\frac{\hat{\theta}_{2} z_{2}}{\delta_{2}}\right), \\
\dot{\lambda}_{2}= & \Gamma_{21}\left[\frac{1}{4 \gamma_{2}^{2}} \lambda_{2} \xi_{2}^{T} \xi_{2} z_{2}^{2}-\sigma_{21}\left(\lambda_{2}-\lambda_{2}^{0}\right)\right], \\
\dot{\hat{\theta}}_{2}= & \Gamma_{22}\left[\left\|z_{2}\right\|-\sigma_{22}\left(\hat{\theta}_{2}-\theta_{2}^{0}\right)\right] .
\end{aligned}
$$

Design parameters in the controller and adaptation laws are chosen as $k_{1}=0.2, k_{2}=0.8, \gamma_{1}=1, \gamma_{2}=2$, $\mu=0.9, \Gamma_{11}=1, \Gamma_{12}=1, \Gamma_{21}=1, \Gamma_{22}=1, \delta_{1}=$ $0.01, \delta_{2}=0.02, \lambda_{1}^{0}=0, \lambda_{2}^{0}=0, \theta_{1}^{0}=0, \theta_{2}^{0}=0$, $\sigma_{11}=0.01, \sigma_{12}=0.01, \sigma_{21}=0.01, \sigma_{22}=0.01$ if the initial conditions are given as $x_{1}(0)=0.12, x_{2}(0)=0$, $\lambda_{1}(0)=0, \lambda_{2}(0)=0, \hat{\theta}_{1}(0)=0, \hat{\theta}_{2}(0)=0$.

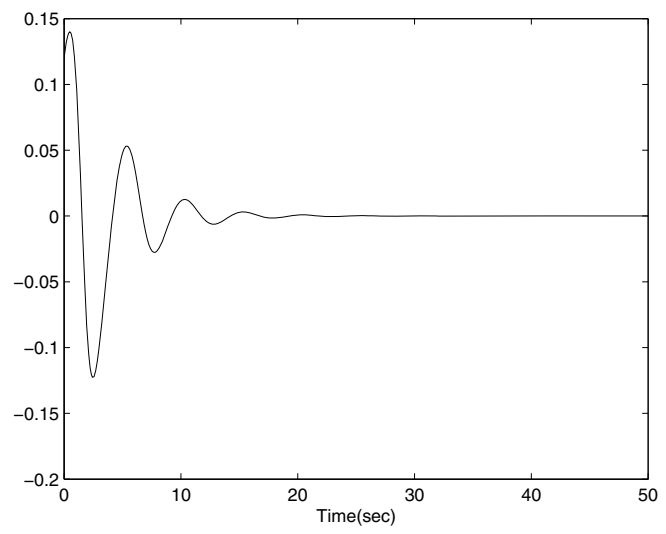

Fig. 4. Trajectory of $x_{1}$.

The simulation results are shown in Figs. 4-6, where Figs. 4 and 5 show the trajectories of $x_{1}, x_{2}$, respectively, while Fig. 6 shows the trajectory of control input $u$. From the above simulation results, it is concluded that the adaptive fuzzy control method (Yang et al., 2005) can 


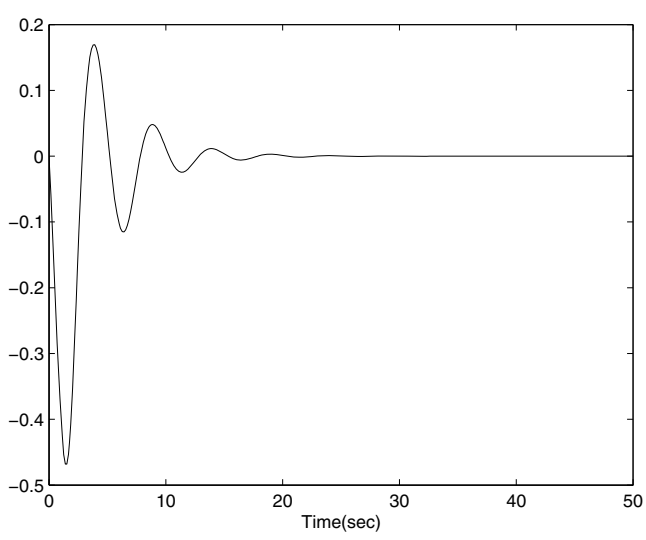

Fig. 5. Trajectory of $x_{2}$.

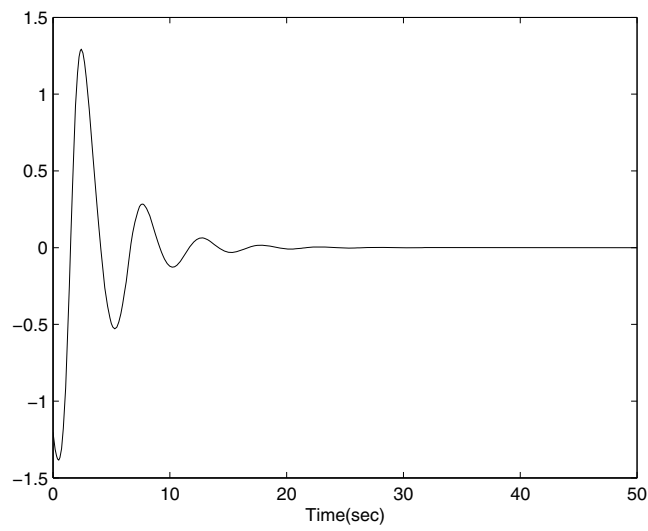

Fig. 6. System control input $u$.

guarantee that all variables of the closed-loop systems are bounded and can achieve better control performance under the assumptions that the controlled nonlinear system does not contain unmodeled dynamics or dynamical disturbances, and the states are measured directly.

Case 2: Consider the nonlinear system (129) with unmodeled dynamics and dynamical disturbances, and assume that $x_{1}$ and $x_{2}$ are both measured.

For this case, use the same control scheme and the initial conditions as in Case 1, and we obtain the simulation results, which are shown by Figs. 7-8. From these one can conclude that the control scheme of Yang et al. (2005) cannot guarantee that the variables $x_{1}, x_{2}$ and $u$ are bounded if the nonlinear system considered contains unmodeled dynamics and dynamical disturbances. The above simulation results in Example 1 and the simulation comparison in Example 2 demonstrate that the proposed adaptive fuzzy control approach can guarantee that all the signals in the closed-loop system are uniformly ultimately bounded and achieve better control performance even if the controlled system contains unmeasured states, unmodeled dynamics and dynamical disturbances.

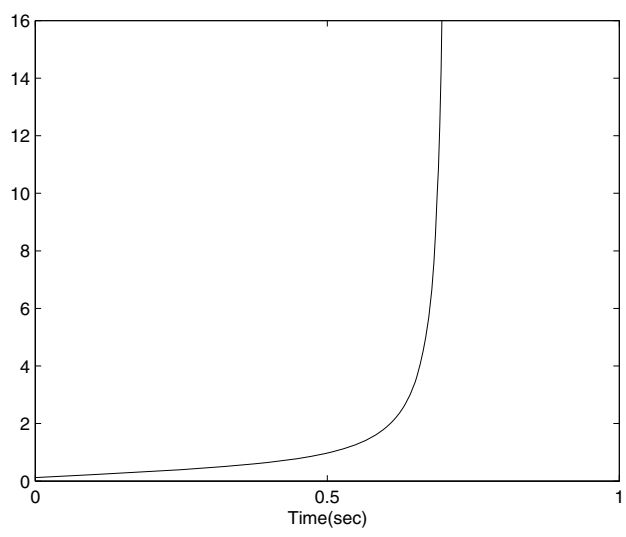

Fig. 7. Trajectory of $x_{1}$.

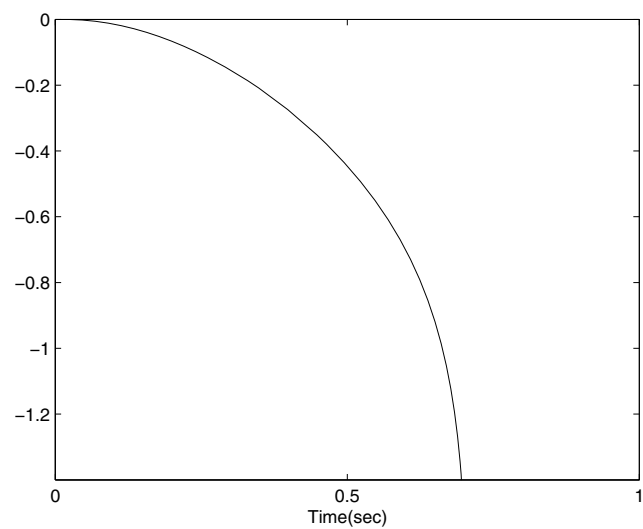

Fig. 8. Trajectory of $x_{2}$.

\section{Conclusion}

In this paper, an adaptive fuzzy output feedback robust control approach was developed for a class of SISO strictfeedback nonlinear systems by combining backstepping design, K-filters and a small-gain theorem. The proposed control approach not only guarantees that all variables of the closed-loop system are uniformly ultimately bounded, but it also has a strong robustness to unmodeled dynamics and dynamical disturbances. Meanwhile, it cancels the restrictive condition given in recent works (Yang et al., 2005; Tong et al., 2010a; 2010b) that the states of controlled systems must be measured directly. Therefore, this paper has extended the existing results for the adaptive fuzzy backstepping control to nonlinear systems.

\section{Acknowledgment}

This work was supported by the National Natural Science Foundation of China (No. 61074014), the Outstanding Youth Funds of the Liaoning Province (No. 2005219001) and the Educational Department of the Liaoning Province (No. 2006R29 and No. 2007T80). 


\section{References}

Boukezzoula, R., Galichet S.and Foulloy L. (2007). Fuzzy feedback linearizing controller and its equivalence with the fuzzy nonlinear internal model control structure, International Journal of Applied Mathematics and Computer Science 17(2): 233-248, DOI: 10.2478/v10006-007-0021-4.

Chen, B. and Liu, X.P. (2005). Fuzzy approximate disturbance decoupling of MIMO nonlinear systems by backstepping and application to chemical processes, IEEE Transactions on Fuzzy Systems 13(6): 832-847.

Chen, B.and Liu, X.P. (2007). Fuzzy approximate disturbance decoupling of MIMO nonlinear systems by backstepping approach, Fuzzy Sets and Systems 158(10): 1097-1125.

Chen, B.S., Lee C.H. and Chang, Y.C. (1996). $H^{\infty}$ tracking design of uncertain nonlinear SISO systems: Adaptive fuzzy approach, IEEE Transactions on Fuzzy Systems 4(1): 3243.

Coddington, E.A. (1989). An Introduction to Ordinary Differential Equations, Prentice-Hall, Englewood Cliffs, NJ.

Denai, M.A. and Attia S.A. (2002). Fuzzy and neural control of an induction motor, International Journal of Applied Mathematics and Computer Science 12(2): 221-233.

Jiang, Z.P., Marels, I.M.Y. and Wang, Y (1996). A Lyapunov formulation of the nonlinear small-gain theorem for interconnected ISS systems, Automatica 32(8): 1211-1215.

Jiang, Z.P. and Praly L. (1998). Design of robust adaptive controllers for nonlinear systems with dynamic uncertainties, Automatica 34(7): 825-840.

Jiang, Z.P. (1999). A combined backstepping and small-gain approach to adaptive output feedback control, Automatica 35(6): 1131-1139.

Kanellakopopoulos, I., Kokotovic, P.V. and Morse, A.S. (1991). Systematic design of adaptive controllers for feedback linearizable systems, IEEE Transactions on Automatic Control 36(11): 1241-1253.

Kristic, M., Kanellakopoulos, I. and Kokotovic, P.V. (1992). Adaptive nonlinear control without over parametrization, System Control Letters 19(3): 177-185.

Kristic, M., Kanellakopoulos, I.and Kokotovic, P.V. (1995). Nonlinear and Adaptive Control Design, Wiley, New York, NY.

Qi, R.Y. and Brdys M.A.(2009). Indirect adaptive controller based on a self-structuring fuzzy system for nonlinear modeling and control, International Journal of Applied Mathematics and Computer Science 19(4): 619-630, DOI: 10.2478/v10006-009-0049-8.

Qian, C.J. and Lin W. (2002). Output feedback control of a class of nonlinear systems: A non-separation principle paradigm, IEEE Transactions on Automatic Control 47(10): 1710-1715.

Tong, S.C., He, X.L. and Li, Y.M. (2010a). Direct adaptive fuzzy backstepping robust control for single input single output uncertain nonlinear systems with small-gain approach, Information Sciences 180(9): 1738-1758.
Tong, S.C., He, X.L. and Li, Y.M. (2010b). Adaptive fuzzy backstepping robust control for uncertain nonlinear systems based on small-gain approach, Fuzzy Sets and Systems 161(6): 771-796.

Wang, L.X. (1994). Adaptive Fuzzy Systems and Control: Design and Stability Analysis, Prentice-Hall, Englewood Cliffs, NJ.

Wang, M., Chen, B., Liu, X.P. and Shi, P. (2007). Adaptive fuzzy tracking control for a class of perturbed strictfeedback nonlinear time-delay systems, Fuzzy Sets and Systems 159(8): 949-967.

Yang, Y.S. and Zhou, C.J.(2005). Robust adaptive fuzzy tracking control for a class of perturbed strict-feedback nonlinear systems via small-gain approach, Information Sciences 170(2-4): 211-234.

Ye, X. D. (2001). Adaptive nonlinear output-feedback control with unknown high-frequency gain sign, IEEE Transactions on Automatic Control 51(3): 504-511.

Zou, A.M. and Hou Z.G. (2008). Adaptive control of a class of nonlinear pure-feedback systems using fuzzy backstepping approach, IEEE Transactions on Fuzzy Systems 16(4): 886-867.

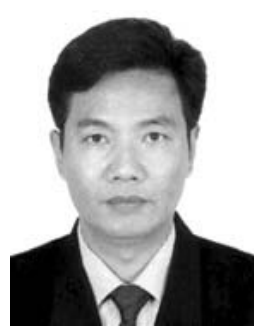

Shaocheng Tong received the B.Sc. degree in mathematics from Jinzhou Normal College, Jinzhou, China, the M.Sc. degree in fuzzy mathematics from Dalian Marine University, Dalian, China, and the Ph.D. degree in fuzzy control from Northeastern University, Shenyang, China, in 1982, 1988, and 1997, respectively. He is currently a professor with the Department of $\mathrm{Ba}$ sic Mathematics, Liaoning University of Technology, Jinzhou, China. His current research interests include fuzzy control theory, nonlinear adaptive control, and intelligent control.

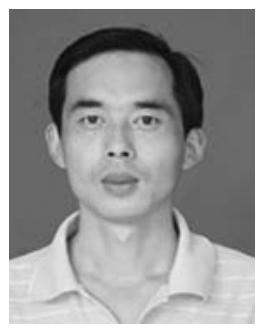

Changliang Liu graduated with a major in mathematics education and applied mathematics from Shandong Education College, Ji'nan, China, in 2003. He is currently working toward the M.Sc. degree with a major in control theory and control engineering at the Liaoning University of Technology, Jinzhou, China. His current research interests include fuzzy control theory, nonlinear adaptive control, decentralized control, and intelligent control.

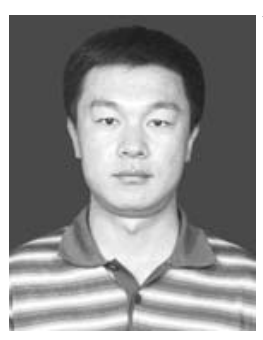

Yongming $\mathbf{L i}$ received the B.Sc. and M.Sc. degrees in applied mathematics from the Liaoning University of Technology, Jinzhou, China, in 2004 and 2007, respectively. He is currently a lecturer at the Department of Basic Mathematics, of the same university. His current research interests include fuzzy control theory, nonlinear adaptive control, and intelligent control.

Received: 2 March 2010

Revised: 1 September 2010

Re-revised: 26 September 2010 